\title{
BIOINDICATION OF WATER PROPERTIES BY ALGAL COMMUNITIES IN THE PAMIR HIGH MOUNTAIN MINERAL AND THERMAL SPRINGS
}

\author{
Toirbek NIYATBEKOV* and Sophia BARINOVA ** \\ * Institute of Botany, Plant Physiology and Genetics, Karamov Street 27, Dushanbe, Tajikistan, \\ TJ-734017, tohir-73@mail.ru \\ ** University of Haifa, Institute of Evolution, Mount Carmel, Abba Khoushi Avenue 199, Haifa, Israel, \\ IL-3498838, Israel, sophia@evo.haifa.ac.il
}

DOI: 10.2478/trser-2018-0016

KEYWORDS: diatoms, thermal, mineral springs, Pamir, Tajikistan.

\section{ABSTRACT}

The bioindication methods have been implemented for the first time for the analyses of water properties in six mineral and thermal springs of Pamir. The studied environment was characterized as flow-alkaline, well oxygenated with low salinity, and low to middle organically polluted. Studied diatom communities preferred periphytonic and benthic habitats of temperate temperature waters. Bioindicator species preferred photosynthetic way of protein synthesis. In first time assessed the trophic level of each studied source show that all they have a state from oligotrophic to mesotrophic. Bioindication methods revealed the effectiveness of nature conservation system in Tajikistan and relevance of bioindication methods using in purpose of assessment and monitoring of water sources on the protected territories.

ZUSAMMENFASSUNG: Bioindikation von Wassereigenschaften durch Algengemeinschaften in den Pamir-Hochgebirgsmineral- und Thermalquellen.

Erstmals wurden für die Analyse der Gewässergüte in sechs Mineral- und Thermalquellen im Pamir Gebiet Bioindikationsmethoden eingesetzt. Die Umgebung der untersuchten Quellen wurde als schwach alkalisch, sauerstoffreich, salzarm und gering bis mittel-organisch eingestuft. Die untersuchten Kieselalgengemeinschaften bevorzugten periphytonische und benthische Habitate von Gewässern mit gemäßigter Temperatur. Die Bioindikator-Arten bevorzugten die photosynthetische Art der Proteinsynthese. Beim ersten Mal zeigte das trophische Niveau jeder untersuchten Quelle einen oligotrophen bis mesotrophen Zustand an. Die Bioindikationsmethoden verdeutlichen die Wirksamkeit des Naturschutzsystems in Tadschikistan sowie die Relevanz von Bioindikationsmethoden bei der Bewertung und Überwachung von Wasserquellen in den geschützten Gebieten.

REZUMAT: Bioindicarea proprietăţilor apei de către comunitățile de alge din izvoarele minerale și termale din munții Pamir.

Metoda bioindicatorilor a fost aplicată pentru prima dată pentru analiza proprietăților apei în șase izvoare minerale și termale din Pamir. Mediul surselor de apă studiate se caracterizează prin alcalinitate slabă, oxigenare bună, salinitate scăzută și poluare organică redusă până la moderată. Comunitățile de diatomee studiate preferă habitatele perifitice și bentonice ale apelor cu temperatură moderată. Speciile bioindicatoare sunt fotosintetizatoare. Pentru prima dată s-a evaluat nivelul trofic al fiecărei surse de apă studiate, constatându-se că acestea sunt de la oligotrofe până la mezotrofe. Metoda bioindicatorilor a evidențiat eficacitatea sistemului de conservare a naturii în Tadjikistan și relevanța folosirii acestei metode în scopul evaluării și monitorizării surselor de apă din ariile protejate. 


\section{INTRODUCTION}

Mineral and thermal springs are widely distributed throughout the world but are most numerous in mountain areas in which there has been volcanic activity in late geologic time (Waring, 1965).

Mineral and thermal springs represent natural waters that are characteristic of the region. Biota in natural high mountain sources has been mostly studied from the middle period of 20th century (Winterbourn, 1969) when most attention was given to cyanobacteria, and only in last years to diatoms also (Fránková et al., 2009; Leira et al., 2017).

Algal species richness and occurrence up to now are studied not enough in mountain springs because their usually placed in hard-to-reach areas (Sisma-Ventura et al., 2010).

There are known many springs in Pamir with different water properties (Waring, 1965). The natural springs in Tajikistan are under protection (Bokhodjaev and Davlatmamadov, 1994) and can represent some natural environment as reference characteristics in the context of an increase in the anthropogenic load of the surrounding areas. But study of algal diversity in Pamir springs is in initial stage (Churshina, 1982; Barinova and Niyatbekov, 2017) although its algal species ecology can help to characterize the reference natural water properties in this important and interesting area of the world.

Freshwater algae are widely used in ecological assessment of water quality. It is very important to know about algal diversity in inland waters because most algal species can be used as environmental indicators. (Barinova et al., 2006; Sinitean and Kutaşi, 2012; Torrisi and Dell'uomo, 2012; Barinova and Niyatbekov, 2017)

Diversity of algae in Tajikistan has been studied sporadically during the last century. The uppermost part of Tajikistan territory is Pamir where large regional rivers as Panj and Gunt are started. This high mountain area is very rich in thermal and mineral waters. In these waters, for many centuries, a special community of algae with a specific species composition and degree of species resistance to peculiarly extreme environmental conditions was formed and developed. Therefore, Pamir is one of high altitude area in Eurasia with close relations to Hindu Cush, Altay, and Himalayas. Its territory has diverse aquatic habitats from clear freshwater large rivers, streams, lakes, to mineral and thermal springs which are occupied by diverse algal communities.

Our own study of diatoms in thermal and mineral springs was enriched by the regional diversity (Barinova and Niyatbekov, 2017) but we assume that the diversity study of this group of algae in Pamir is still far from complete. Nevertheless, it can help to characterize the high mountain aquatic habitats water quality by bio-indication methods.

Thus, the aim of our work was to reveal species-specific ecological preferences of diatom algae from studied mineral and thermal springs of Pamir to assess its water quality.

Pamir is very rich in thermal and mineral waters, which in a way are unique habitats characterized by a constantly and high temperature from $10^{\circ} \mathrm{C}$ to $86^{\circ} \mathrm{C}$ and various chemical compositions saturated with carbon dioxide and nitrogen gases such as hydrogen sulfidesiliceous, hydrocarbonate-sulphate-calcium-magnesium, chloride-sulfate-calcium-sodium, hydrocarbonate-sulfate-sodium and weak radon-chloride-sulfate (Churshina, 1982; Bokhodjaev and Davlatmamadov, 1994). In these waters, for many centuries, a special community of algae with a specific species composition was formed. 


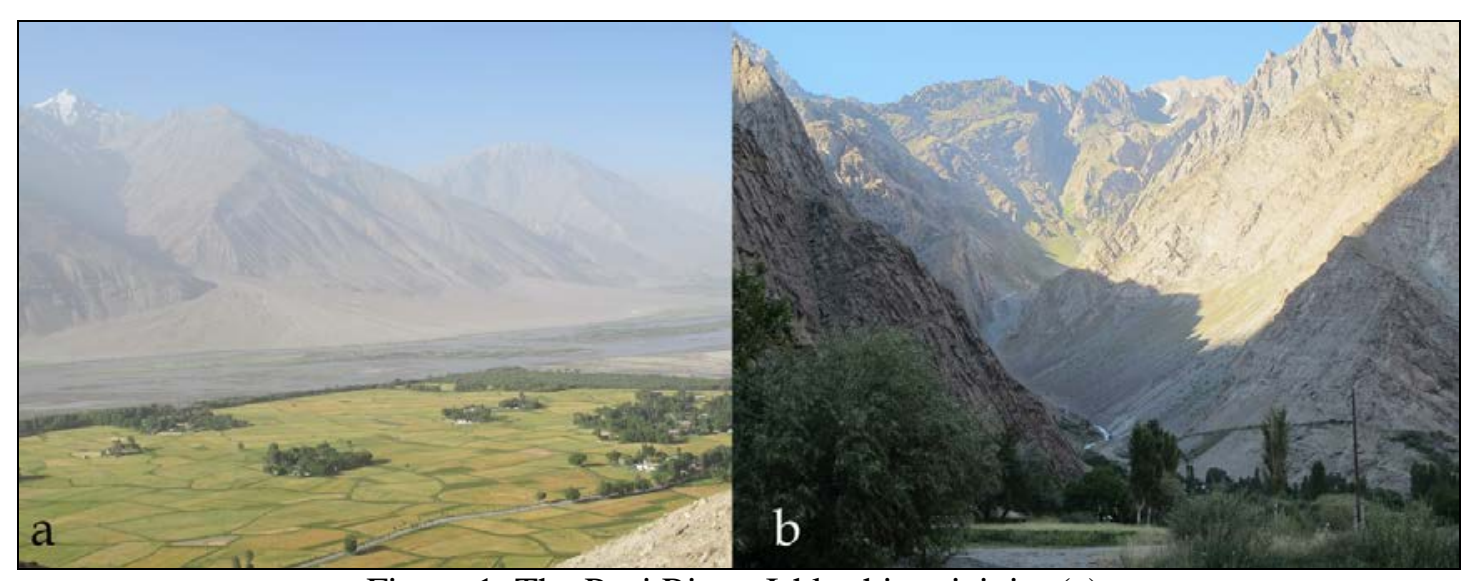

Figure 1: The Panj River, Ishkashim vicinity (a); the Bartang Canyon (b).

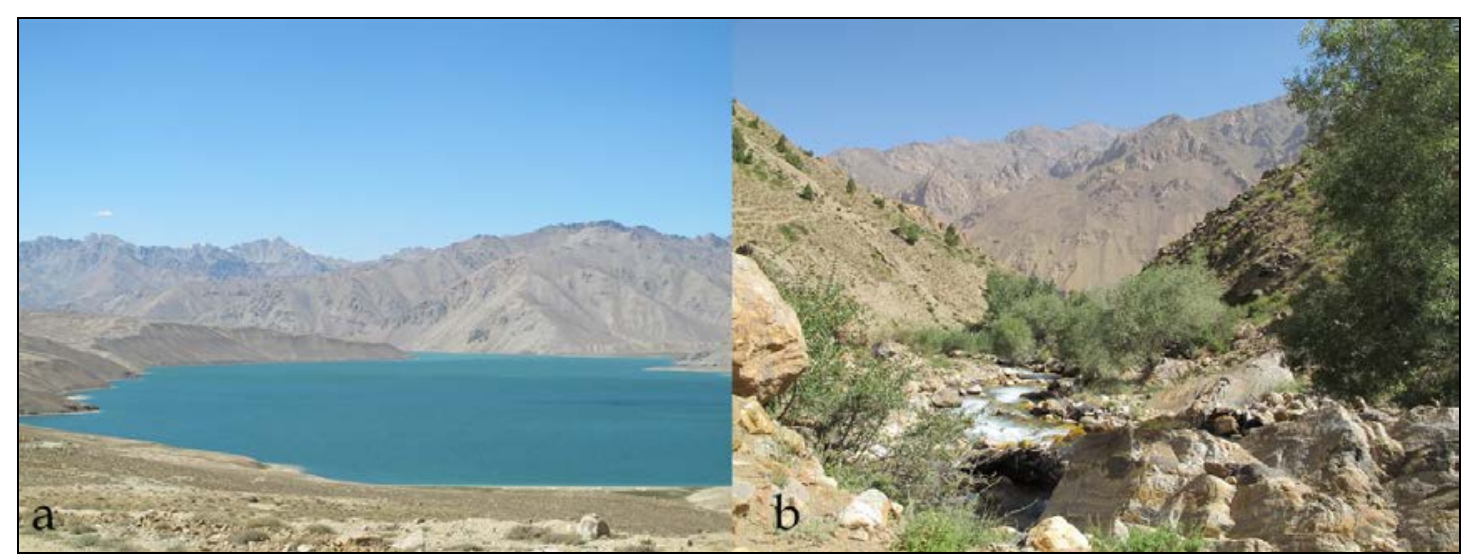

Figure 2: The Yashilkul Lake and its vicinity (a); the Yazgulam Canyon (b).

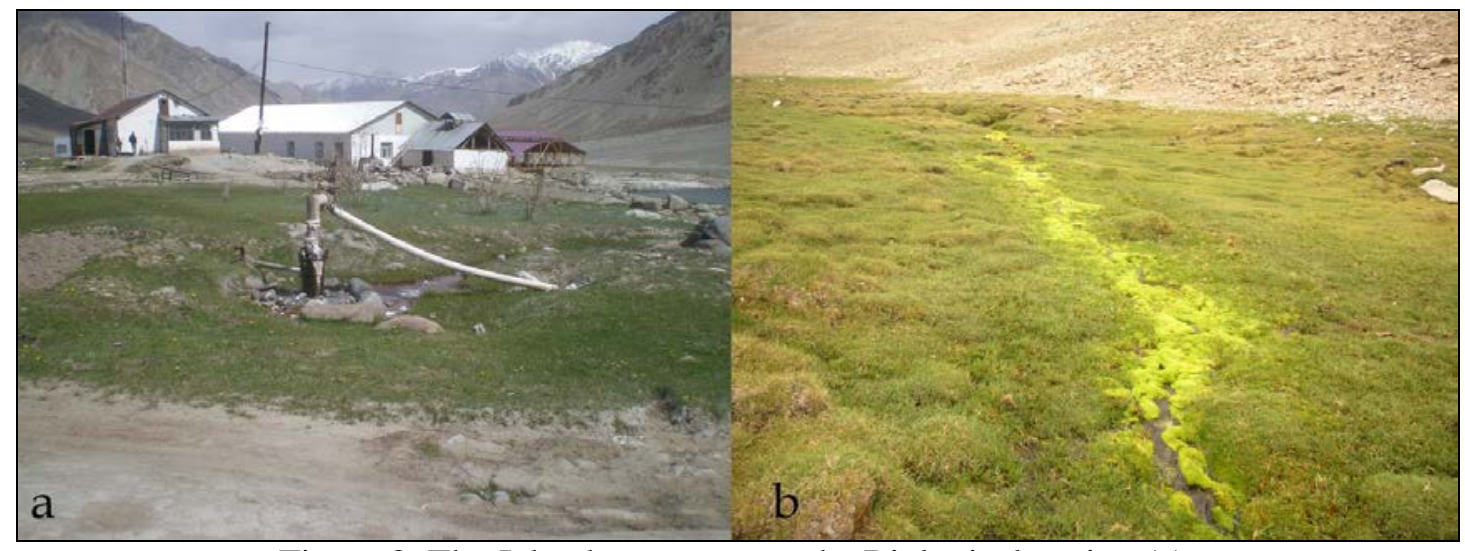

Figure 3: The Jelandy source near the Biological station (a); the Jelandy source vicinity (b). 


\section{MATERIAL AND METHODS}

The biological material for this study is represented by personal data (150 samples collected in the summer of 2000-2015 from Jelandy, Avadzh, Garm-Chashma, Sassykbulak, Sist and Barshor located at an altitude from 2,360 to 3,800 m a.s.l. (Figs. 1-3). Algal periphytonic samples were collected and processed from various places, springs, and griffins with a water temperature of $10^{\circ} \mathrm{C}$ to $86^{\circ} \mathrm{C}$ (Tab. 1).

Species ecology data come from database (Barinova et al., 2006) for nine ecological bioindication systems. A total list of revealed diatoms in Pamir mineral and hot springs was correlated with an ecological database in the Microsoft Access Program.

Table 1: Diatom indicator taxa and the major variables of habitats in the mineral water springs of Pamir with geographical coordinates.

\begin{tabular}{|c|c|c|c|c|c|c|}
\hline Pamir region & East & West & West & East & West & West \\
\hline $\begin{array}{c}\text { Name of } \\
\text { spring }\end{array}$ & Jelandy & Avadzh & $\begin{array}{c}\text { Garm- } \\
\text { Chashma }\end{array}$ & Sassykbulak & Sist & Barshor \\
\hline $\begin{array}{c}\text { No. of } \\
\text { spring }\end{array}$ & 1 & 2 & 3 & 4 & 5 & 6 \\
\hline $\begin{array}{c}\text { No. of } \\
\text { indicator taxa }\end{array}$ & 92 & 48 & 18 & 14 & 9 & 18 \\
\hline $\begin{array}{c}\text { Altitude, } \\
\text { m a.s.l. }\end{array}$ & 3,600 & 2,410 & 2,800 & 3,800 & 2,360 & 2,400 \\
\hline $\begin{array}{c}\text { Temperature, } \\
{ }^{\circ} \mathrm{C}\end{array}$ & $21-86$ & 35 & $40-62$ & 22 & $10-12$ & $10-15$ \\
\hline pH & 7.8 & 7.1 & 7.1 & 7.4 & 5.8 & 6.4 \\
\hline North & $37^{\circ} 34^{\prime}$ & $\begin{array}{l}37^{\circ} 12^{\prime} \\
19.06^{\prime}\end{array}$ & $\begin{array}{c}37^{\circ} 12^{\prime} \\
11.06^{\prime}\end{array}$ & $\begin{array}{c}37^{\circ} 40^{\prime} \\
84.90^{\prime}\end{array}$ & $\begin{array}{c}37^{\circ} 10^{\prime} \\
18.26^{\prime}\end{array}$ & $\begin{array}{c}37^{\circ} 00^{\prime} \\
98.02^{\prime}\end{array}$ \\
\hline East & $\begin{array}{c}72^{\circ} 34^{\prime} \\
41.93^{\prime}\end{array}$ & $\begin{array}{c}71^{\circ} 31^{\prime} \\
90.51^{\prime}\end{array}$ & $\begin{array}{c}71^{\circ} 32^{\prime} \\
11.18^{\prime}\end{array}$ & $\begin{array}{c}73^{\circ} 00^{\prime} \\
22.54^{\prime}\end{array}$ & $\begin{array}{c}71^{\circ} 30^{\prime} \\
19.87^{\prime}\end{array}$ & $\begin{array}{c}71^{\circ} 30^{\prime} \\
21.89^{\prime}\end{array}$ \\
\hline
\end{tabular}

RESULTS AND DISCUSSION

Data about studied communities from six sources are represented in table 1 with relation to the West or East Pamir regions. We calculated Pearson coefficients for species richness, water $\mathrm{pH}$, temperature and altitude of source with wessa.net and find that only water $\mathrm{pH}$ and altitude have significant correlation 0.78 with $\mathrm{p}<0.03$.Alkaline sources are placed in highest altitude and acidic-water sources are in altitude about 2,400 m a.s.l. (Fig. 4). Water temperature in the studied sources is varied even in the same source, but uppermost localities have highest water temperature.

As a result of laboratory processing of 150 periphytonic samples from six thermal and mineral springs, the regional diversity was enriched by 134 diatom species (166 with infraspecific taxa) (Barinova and Niyatbekov, 2017). For the basic list of species, we revealed species-specific ecological properties that are represented in table 2. All found taxa were indicators of substrate preferences, oxygenation, water $\mathrm{pH}$, chlorides concentration, organic pollution, nutrition type, and trophic level.

Species richness of diatoms in the studied source communities is varied in the broad range from 92 in Jelandy to 9 in Sist. Table 1 and figure 4 shows that highest diversity was found in the studied sources with alkaline water with highest temperature. Remarkably, the Jelandy source, where water is $7.8 \mathrm{pH}$ and temperature varied in the range $21-86^{\circ} \mathrm{C}$, it was revealed that maximal species richness was -92 taxa. 
Table 2: Diversity and ecology of diatom algae in the thermal and mineral water sources of Pamir with species autecology. Number of springs 1-6 as in table 1.

\begin{tabular}{|c|c|c|c|c|c|c|c|c|c|c|c|c|c|c|c|}
\hline Таха & - & $\sim$ & $m$ & $\nabla$ & L & 0 & ত్రి & $H$ & ठิ & 吾 & 苂 & D & लే & $\stackrel{8}{9}$ & 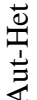 \\
\hline $\begin{array}{l}\text { Achnanthes } \\
\text { exigua } \\
\text { Grunow in } \\
\text { Cleve and } \\
\text { Grunow }\end{array}$ & - & 1 & 1 & 1 & 1 & 1 & $\infty$ & 㤩 & $\begin{array}{l}\frac{v}{p} \\
\frac{1}{\omega}\end{array}$ & $\frac{\Psi}{\pi}$ & $-r$ & के & $\stackrel{\circ}{\mathrm{i}}$ & $\begin{array}{l}0 \\
\vdots\end{array}$ & 竞 \\
\hline $\begin{array}{l}\text { Achnanthes } \\
\text { gibberula } \\
\text { Grunow }\end{array}$ & - & 1 & - & -1 & 1 & 1 & $m$ & 莺 & $\begin{array}{l}\frac{5}{5} \\
\frac{1}{\omega n}\end{array}$ & 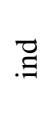 & $\overline{\mathrm{E}}$ & ฝ & mạ & ఫ్ & 1 \\
\hline $\begin{array}{l}\text { Achnanthes } \\
\text { Gibberula } \\
\text { var. interrupta } \\
\text { Poretzky and } \\
\text { Anisimova }\end{array}$ & -1 & 1 & 1 & 1 & 1 & 1 & $\infty$ & 壳 & $\begin{array}{l}\frac{v}{p} \\
\frac{1}{\omega}\end{array}$ &.$\Xi$ & 司 & ฝ & mo & ఫ̄ & 1 \\
\hline $\begin{array}{l}\text { Achnanthidium } \\
\text { minutissimum } \\
\text { (Kützing) } \\
\text { Czarnecki }\end{array}$ & - & 1 & 1 & 1 & 1 & 1 & $\stackrel{m}{a}$ & $\begin{array}{l}\text { 䍖 } \\
\end{array}$ & $\begin{array}{l}\frac{1}{4} \\
\frac{1}{\omega}\end{array}$ & 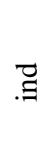 & $r$ & $\approx$ & $\begin{array}{l}\text { 号 } \\
0\end{array}$ & $\begin{array}{l}0 \\
1 \\
0\end{array}$ & $\frac{\mathscr{v}}{\pi}$ \\
\hline $\begin{array}{l}\text { Achnanthidium } \\
\text { thermal } \\
\text { Rabenhorst }\end{array}$ & - & 1 & 1 & I & 1 & 1 & $\infty$ & 志 & $\begin{array}{l}\frac{ \pm}{5} \\
\frac{1}{\omega}\end{array}$ & 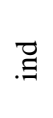 & हี & $\stackrel{x}{n}$ & חִ & 疋 & 1 \\
\hline $\begin{array}{l}\text { Actinella } \\
\text { punctata } \\
\text { Lewis F. W. }\end{array}$ & -1 & 1 & 1 & I & 1 & I & 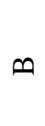 & 1 & 1 & $\ddot{\widetilde{\sigma}}$ & 로 & 1 & $\stackrel{-}{\circ}$ & t & 1 \\
\hline $\begin{array}{l}\text { Amphora } \\
\text { ovalis } \\
\text { (Kützing) } \\
\text { Kützing }\end{array}$ & - & 1 & $\neg$ & I & 1 & 1 & $m$ & 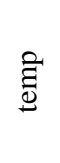 & $\begin{array}{l}\frac{n}{p} \\
\frac{p}{\omega}\end{array}$ & సै & -7 & ঝ & น? & $\stackrel{\Xi}{E}$ & $\frac{\tilde{\sigma}}{\pi}$ \\
\hline $\begin{array}{l}\text { Amphora } \\
\text { ovalis } \\
\text { var. ediculus } \\
\text { (Kützing) } \\
\text { Van Heurck }\end{array}$ & 1 & -1 & 1 & I & 1 & 1 & $\infty$ & 哀 & $\vec{w}$ & गै & $r$ & 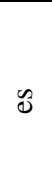 & $\hat{-}$ & 录 & $\frac{8}{\pi}$ \\
\hline
\end{tabular}


Table 2 (continued): Diversity and ecology of diatom algae in the thermal and mineral water sources of Pamir with species autecology. Number of springs 1-6 as in table 1.

\begin{tabular}{|c|c|c|c|c|c|c|c|c|c|c|c|c|c|c|c|}
\hline Таха & - & $N$ & $m$ & $\nabla$ & L & 0 & 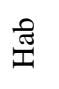 & $H$ & ôे & 垔 & 胥 & D & जే & 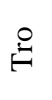 & $\begin{array}{l}\text { 离 } \\
\end{array}$ \\
\hline $\begin{array}{l}\text { Amphora } \\
\text { ovalis } \\
\text { var. lybica } \\
\text { (Ehrenberg) } \\
\text { Cleve }\end{array}$ & 1 & $\neg$ & 1 & 1 & 1 & 1 & $\infty$ & 宅 & $\ddot{\omega}$ & त्ञ & $\cdot r$ & 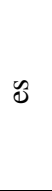 & $\stackrel{n}{-}$ & 录 & 1 \\
\hline $\begin{array}{l}\text { Aneumastus } \\
\text { minor } \\
\text { Lange- } \\
\text { Bertalot }\end{array}$ & -1 & 1 & 1 & 1 & 1 & 1 & 竞 & 1 & 1 & $\frac{W}{\sigma}$ & $-r$ & 1 & $\stackrel{\text { Iִ }}{\rightarrow}$ & ڤ్ & 1 \\
\hline $\begin{array}{l}\text { Aneumastus } \\
\text { rostratus } \\
\text { (Hustedt) } \\
\text { Lange- } \\
\text { Bertalot }\end{array}$ & 1 & 1 & 1 & -1 & 1 & 1 & $\stackrel{m}{a}$ & 1 & 1 & 㫕 & $\cdot r$ & 1 & $\stackrel{\circ}{\mathrm{i}}$ & 1 & 1 \\
\hline $\begin{array}{l}\text { Aneumastus } \\
\text { tuscula } \\
\text { f. intermedia } \\
\text { Kisselev }\end{array}$ & -1 & 1 & 1 & 1 & 1 & 1 & 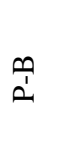 & 1 & 1 & 㫕 & $\cdot r$ & 1 & $\stackrel{\sigma}{0}$ & $\begin{array}{l}0 \\
\text { İ }\end{array}$ & 1 \\
\hline $\begin{array}{l}\text { Aulacoseira } \\
\text { italica } \\
\text { (Ehrenberg) } \\
\text { Simonsen }\end{array}$ & -1 & $\neg$ & 1 & | & 1 & 1 & $\begin{array}{l}m \\
\vdots \\
a\end{array}$ & $\overrightarrow{0}$ & $\begin{array}{l}\frac{\vec{n}}{\omega} \\
\frac{1}{\omega}\end{array}$ & 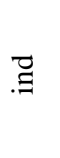 & $\cdot r$ & $\approx$ & $\stackrel{\text { If }}{\rightarrow}$ & 苂 & 1 \\
\hline $\begin{array}{l}\text { Brachysira } \\
\text { serians } \\
\text { (Brébisson) } \\
\text { Round and } \\
\text { Mann D. G. }\end{array}$ & -1 & 1 & 1 & | & 1 & 1 & $\infty$ & 1 & $\begin{array}{l}\frac{\vec{v}}{\omega} \\
\frac{1}{\omega}\end{array}$ & $\underset{\widetilde{U}}{\tilde{U}}$ & ᄅ & 1 & ก̃ & t & $\frac{\mathscr{y}}{\tilde{\sigma}}$ \\
\hline $\begin{array}{l}\text { Caloneis } \\
\text { bacillum } \\
\text { (Grunow) } \\
\text { Cleve }\end{array}$ & - & 1 & 1 & | & 1 & $\neg$ & $\infty$ & 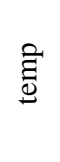 & $\begin{array}{l}\frac{1}{\omega} \\
\dot{1} \\
\omega\end{array}$ & 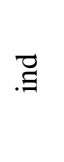 & $-r$ & 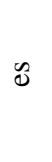 & $\stackrel{M}{-}$ & 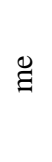 & $\frac{\mathscr{0}}{\tilde{\pi}}$ \\
\hline $\begin{array}{l}\text { Caloneis } \\
\text { silicula } \\
\text { (Ehrenberg) } \\
\text { Cleve }\end{array}$ & -1 & I & 1 & 1 & 1 & 1 & $\infty$ & 1 & $\ddot{\omega}$ & 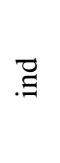 & $-r$ & के & $\stackrel{m}{-}$ & 录 & $\frac{\mathscr{n}}{\pi}$ \\
\hline $\begin{array}{l}\text { Caloneis } \\
\text { silicula var. } \\
\text { kjellmaniana } \\
\text { Cleve }\end{array}$ & -1 & 1 & 1 & 1 & 1 & 1 & $\infty$ & 1 & 1 & ने & $\rightarrow$ & 1 & 1 & 1 & 1 \\
\hline
\end{tabular}


Table 2 (continued): Diversity and ecology of diatom algae in the thermal and mineral water sources of Pamir with species autecology. Number of springs 1-6 as in table 1.

\begin{tabular}{|c|c|c|c|c|c|c|c|c|c|c|c|c|c|c|c|}
\hline Таха & $-r$ & $\sim$ & $m$ & $\nabla$ & L & 0 & 苈 & $H$ & ठิ & 吾 & 苂 & D & लే & $\stackrel{8}{9}$ & 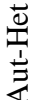 \\
\hline $\begin{array}{l}\text { Cavinula } \\
\text { lacustris } \\
\text { (Gregory W.) } \\
\text { Mann D. G. } \\
\text { and Stickle } \\
\text { in Round, } \\
\text { Crawford } \\
\text { and Mann }\end{array}$ & - & 1 & 1 & 1 & 1 & 1 & $n$ & 1 & 1 & 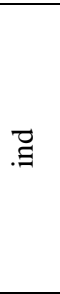 & $r$ & 1 & $\stackrel{\circ}{\circ}$ & 疋 & $\frac{\mathscr{n}}{\pi}$ \\
\hline $\begin{array}{l}\text { Cocconeis } \\
\text { pediculus } \\
\text { Ehrenberg }\end{array}$ & 1 & - & 1 & 1 & 1 & 1 & $n$ & 1 & $\begin{array}{l}\frac{5}{4} \\
\frac{1}{\omega}\end{array}$ & $\frac{W}{\pi}$ & $-r$ & 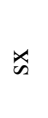 & $\stackrel{\infty}{\rightarrow}$ & 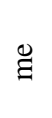 & $\frac{\mathscr{D}}{\pi}$ \\
\hline $\begin{array}{l}\text { Cocconeis } \\
\text { placentula } \\
\text { Ehrenberg }\end{array}$ & - & $\neg$ & 1 & 1 & 1 & 1 & â & 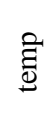 & $\begin{array}{l}\frac{b}{\omega} \\
\frac{1}{\omega}\end{array}$ & $\frac{W}{\pi}$ & $r$ & 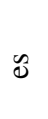 & 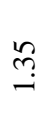 & छั & $\frac{\mathscr{v}}{\pi}$ \\
\hline $\begin{array}{l}\text { Cocconeis } \\
\text { placentula } \\
\text { var. rouxii } \\
\text { (Héribaud- } \\
\text { Joseph } \\
\text { and Brun) } \\
\text { Cleve }\end{array}$ & - & 1 & 1 & I & 1 & 1 & $m$ & 1 & 1 & $\frac{W}{\pi}$ & $r$ & 1 & $\stackrel{\leftrightarrow}{\rightarrow}$ & 1 & 1 \\
\hline $\begin{array}{l}\text { Cocconeis } \\
\text { scutellum } \\
\text { Ehrenberg }\end{array}$ & 1 & -1 & 1 & I & 1 & 1 & $n$ & 1 & 1 & 1 & $I$ & 1 & 1 & 1 & 1 \\
\hline $\begin{array}{l}\text { Craticula } \\
\text { cuspidata } \\
\text { (Kützing) } \\
\text { Mann }\end{array}$ & -1 & 1 & 1 & I & 1 & 1 & $m$ & 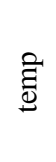 & $\begin{array}{l}\frac{\bar{v}}{4} \\
\frac{1}{\omega}\end{array}$ & $\frac{\vec{U}}{\sigma}$ & $r$ & $\approx$ & $\stackrel{\text { Iq }}{i}$ & 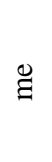 & 1 \\
\hline $\begin{array}{l}\text { Craticula } \\
\text { halophila } \\
\text { (Grunow) } \\
\text { Mann D. G. } \\
\text { in Round, } \\
\text { Crawford } \\
\text { and Mann } \\
\end{array}$ & 1 & - & 1 & I & 1 & 1 & 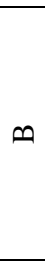 & 1 & $\frac{\bar{p}}{\frac{1}{\omega}}$ & $\overrightarrow{\widetilde{\sigma}}$ & $\bar{\Xi}$ & 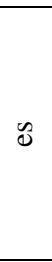 & $\stackrel{\circ}{\dot{m}}$ & 0 & $\frac{\mathscr{g}}{\pi}$ \\
\hline
\end{tabular}


Table 2 (continued): Diversity and ecology of diatom algae in the thermal and mineral water sources of Pamir with species autecology. Number of springs 1-6 as in table 1.

\begin{tabular}{|c|c|c|c|c|c|c|c|c|c|c|c|c|c|c|c|}
\hline Таха & -1 & $N$ & $m$ & $\nabla$ & L & 0 & ত్రి & $H$ & ôे & I & 苂 & D & ज्ञీ & 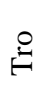 & $\begin{array}{l}\stackrel{\vec{T}}{1} \\
\\
\end{array}$ \\
\hline $\begin{array}{l}\text { Cymbella } \\
\text { affinis } \\
\text { Kützing }\end{array}$ & -1 & 1 & 1 & -1 & 1 & 1 & 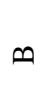 & 宅 & $\begin{array}{l}\frac{ \pm}{5} \\
\frac{1}{\omega}\end{array}$ & 㫕 & $-r$ & ঝ & $\exists$ & t & 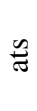 \\
\hline $\begin{array}{l}\text { Cymbella } \\
\text { aspera } \\
\text { (Ehrenberg) } \\
\text { Cleve }\end{array}$ & -1 & 1 & 1 & 1 & 1 & 1 & $\infty$ & 1 & $\begin{array}{l}\frac{ \pm}{5} \\
\frac{1}{\omega}\end{array}$ & $\underset{\Xi}{\Xi}$ & $r$ & 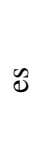 & mo & ò & $\frac{n}{\pi}$ \\
\hline $\begin{array}{l}\text { Cymbella } \\
\text { cistula } \\
\text { (Ehrenberg) } \\
\text { Kirchner O. }\end{array}$ & 1 & -1 & 1 & 1 & 1 & 1 & $\infty$ & 1 & $\begin{array}{l}\frac{v}{p} \\
\frac{p}{\omega}\end{array}$ & 㫕 & $-r$ & $\stackrel{x}{\infty}$ & $\underset{7}{*}$ & 0 & $\frac{\mathscr{n}}{\pi}$ \\
\hline $\begin{array}{l}\text { Cymbella } \\
\text { compacta } \\
\text { Østrup }\end{array}$ & -1 & I & -1 & -1 & 1 & 1 & 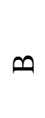 & 1 & 1 & 1 & 1 & 1 & $\hat{0}$ & 1 & 1 \\
\hline $\begin{array}{l}\text { Cymbella } \\
\text { cymbiformis } \\
\text { Agardh C. }\end{array}$ & $-r$ & 1 & 1 & I & 1 & -1 & $\infty$ & 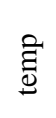 & है &.$\Xi$ & $-r$ & 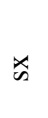 & $\stackrel{\circ}{i}$ & है & $\frac{\mathscr{n}}{\pi}$ \\
\hline $\begin{array}{l}\text { Cymbella } \\
\text { falaisensis } \\
\text { (Grunow) } \\
\text { Krammer and } \\
\text { Lange- } \\
\text { Bertalot } \\
\end{array}$ & 1 & 1 & 1 & I & -1 & 1 & $\infty$ & 1 & के & I & 로 & $\mathscr{\Xi}$ & & ह̦ & $\frac{\mathscr{n}}{\pi}$ \\
\hline $\begin{array}{l}\text { Cymbella } \\
\text { helvetica } \\
\text { Kützing }\end{array}$ & 1 & -1 & I & I & 1 & 1 & $\infty$ & 1 & के &. $\bar{g}$ & $-r$ & 1 & : & 疋 & 1 \\
\hline $\begin{array}{l}\text { Cymbella } \\
\text { helvetica var. } \\
\text { curta Cleve }\end{array}$ & 1 & -1 & 1 & 1 & 1 & 1 & $\infty$ & 1 & 1 & 崩 & 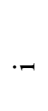 & 1 & 1 & 1 & 1 \\
\hline $\begin{array}{l}\text { Cymbella } \\
\text { hustedtii } \\
\text { Krasske }\end{array}$ & - & 1 & I & I & 1 & 1 & $n$ & 1 & है & $\bar{\Xi}$ & -7 & 1 & $\stackrel{\leftrightarrow}{\circ}$ & Ė & $\frac{n}{\pi}$ \\
\hline
\end{tabular}


Table 2 (continued): Diversity and ecology of diatom algae in the thermal and mineral water sources of Pamir with species autecology. Number of springs 1-6 as in table 1.

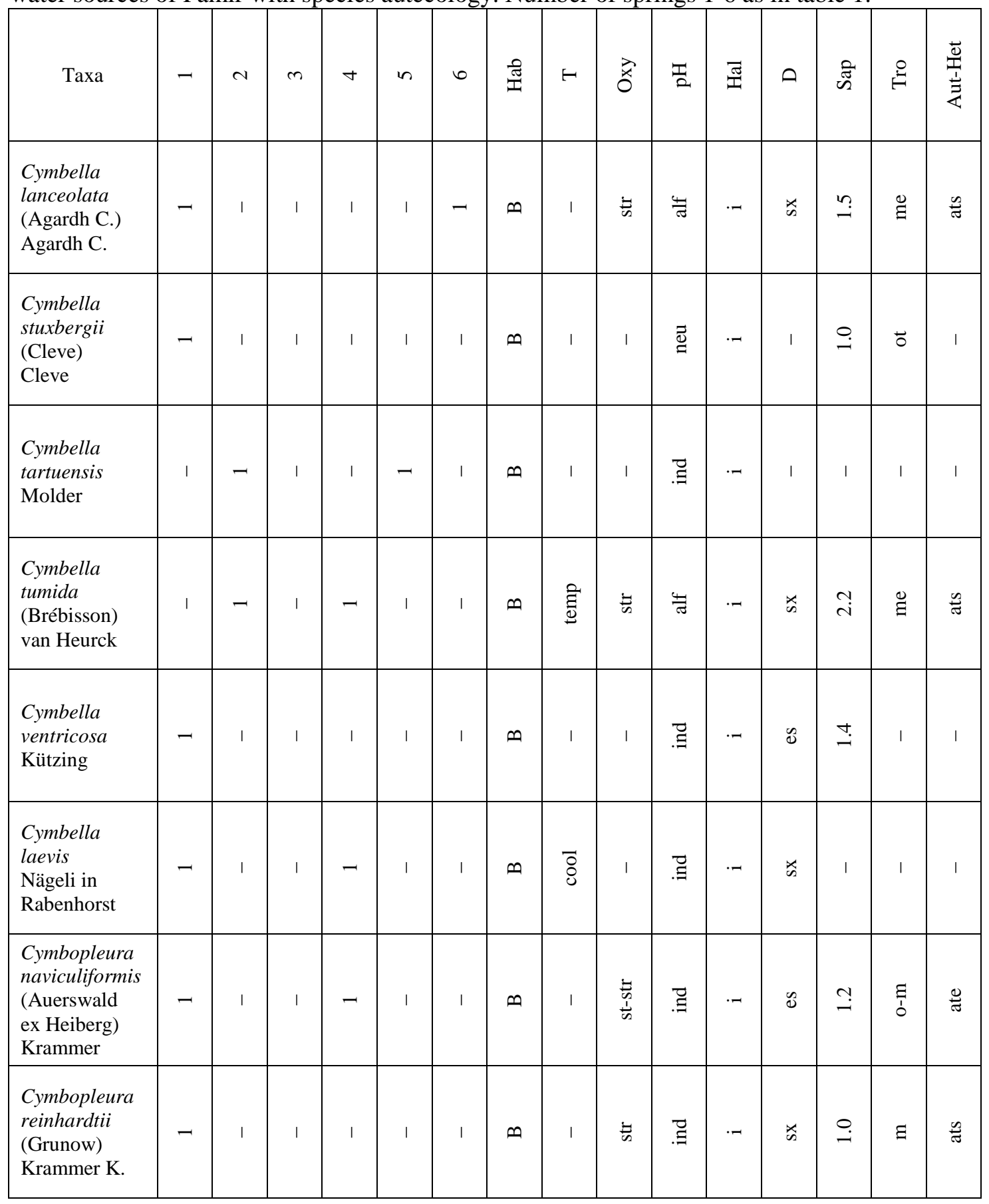


Table 2 (continued): Diversity and ecology of diatom algae in the thermal and mineral water sources of Pamir with species autecology. Number of springs 1-6 as in table 1.

\begin{tabular}{|c|c|c|c|c|c|c|c|c|c|c|c|c|c|c|c|}
\hline Таха & -1 & $N$ & $m$ & $\nabla$ & L & 0 & 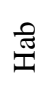 & H & ô & 楶 & 胥 & D & जే & $\stackrel{g}{\Theta}$ & 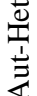 \\
\hline $\begin{array}{l}\text { Denticula } \\
\text { elegans } \\
\text { Kützing }\end{array}$ & 1 & - & 1 & 1 & 1 & 1 & 樆 & 1 & 1 & त्ञ & $-r$ & 1 & பே. & ठ & 1 \\
\hline $\begin{array}{l}\text { Denticula } \\
\text { thermalis } \\
\text { Kützing }\end{array}$ & - & 1 & 1 & 1 & - & 1 & $\infty$ & $\begin{array}{l}\text { 壳 } \\
\text { 3 }\end{array}$ & 1 & 㫕 & 1 & 1 & $\stackrel{\circ}{i}$ & ๕ั & 1 \\
\hline $\begin{array}{l}\text { Diatoma } \\
\text { anceps } \\
\text { (Ehrenberg) } \\
\text { Kirchner }\end{array}$ & - & 1 & 1 & - & 1 & 1 & â & $\overline{8}$ & $\begin{array}{l}\frac{v}{w_{0}} \\
\frac{1}{\omega}\end{array}$ & $\underset{\Xi}{\Xi}$ & ᄅ & w & $\stackrel{0}{0}$ & t & 1 \\
\hline $\begin{array}{l}\text { Diatoma } \\
\text { mesodon } \\
\text { Kützing }\end{array}$ & - & 1 & -1 & 1 & 1 & 1 & $\infty$ & $\overrightarrow{8}$ & $\begin{array}{l}\frac{b}{p} \\
\dot{s} \\
\omega\end{array}$ & $\underset{\Xi}{\tilde{\Xi}}$ & ᄅ & $\stackrel{x}{\infty}$ & $\stackrel{t}{0}$ & t & $\frac{\mathscr{M}}{\pi}$ \\
\hline $\begin{array}{l}\text { Diatoma } \\
\text { vulgaris } \\
\text { Bory }\end{array}$ & - & 1 & 1 & 1 & 1 & 1 & מ̂n & 1 & 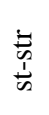 &.$\Xi$ & $-r$ & $\stackrel{x}{n}$ & $\underset{N}{N}$ & 气̆ & 营 \\
\hline $\begin{array}{l}\text { Diatoma } \\
\text { vulgaris } \\
\text { var. linearis } \\
\text { Grunow in } \\
\text { van Heurck }\end{array}$ & - & 1 & 1 & 1 & 1 & 1 & $\infty$ & 1 & के & 㫕 & -7 & $\approx$ & ลี & 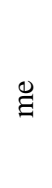 & $\frac{\mathscr{g}}{\pi}$ \\
\hline $\begin{array}{l}\text { Diatoma } \\
\text { vulgaris } \\
\text { var. brevis } \\
\text { Grunow }\end{array}$ & -1 & 1 & 1 & 1 & 1 & 1 & $\stackrel{m}{a}$ & 1 & $\begin{array}{l}\frac{v}{p} \\
\frac{1}{\omega}\end{array}$ & ने & -7 & $\stackrel{\star x}{n}$ & ָ̃ & 气̆ & $\frac{8}{\pi}$ \\
\hline $\begin{array}{l}\text { Didymospheni } \\
\text { a geminata } \\
\text { (Lyngbye) } \\
\text { Schmidt M. }\end{array}$ & - & 1 & -1 & 1 & 1 & 1 & $\infty$ & 1 & 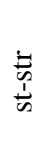 &. $\bar{g}$ & -7 & $\stackrel{x}{n}$ & $\hat{0}$ & $\tilde{o}$ & 1 \\
\hline $\begin{array}{l}\text { Diploneis } \\
\text { ovalis } \\
\text { (Hilse) } \\
\text { Cleve }\end{array}$ & - & 1 & 1 & 1 & 1 & 1 & $\infty$ & 1 & के & तै & -7 & के & g. & है & $\frac{\mathscr{n}}{\pi}$ \\
\hline
\end{tabular}


Table 2 (continued): Diversity and ecology of diatom algae in the thermal and mineral water sources of Pamir with species autecology. Number of springs 1-6 as in table 1.

\begin{tabular}{|c|c|c|c|c|c|c|c|c|c|c|c|c|c|c|c|}
\hline Таха & - & $\sim$ & $m$ & $\nabla$ & เn & 0 & 胥 & $H$ & ô & 壳 & 苂 & D & 芯 & $\stackrel{8}{日}$ & $\begin{array}{l}\text { 离 } \\
\frac{1}{3}\end{array}$ \\
\hline $\begin{array}{l}\text { Diploneis } \\
\text { ovalis var. } \\
\text { oblongella } \\
\text { (Nägeli) } \\
\text { Cleve }\end{array}$ & - & 1 & - & 1 & 1 & 1 & $\infty$ & 1 & $\dot{\xi}$ &.$\Xi$ & $r$ & ঝ & $\ddot{0}$ & ठ & $\frac{\mathscr{v}}{\tilde{\tau}}$ \\
\hline $\begin{array}{l}\text { Ellerbeckia } \\
\text { arenaria } \\
\text { (Moore ex } \\
\text { Ralfs) } \\
\text { Crawford }\end{array}$ & 1 & - & 1 & 1 & 1 & 1 & $\stackrel{m}{a}$ & $\overline{8}$ & $\begin{array}{l}\frac{v}{0} \\
\dot{1} \\
\omega\end{array}$ & $\frac{\Psi}{\sigma}$ & $-r$ & 1 & $\stackrel{0}{0}$ & ठ & 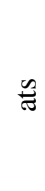 \\
\hline $\begin{array}{l}\text { Encyonema } \\
\text { alpinum } \\
\text { (Grunow) } \\
\text { Mann D. G. } \\
\text { in Round, } \\
\text { Crawford R. } \\
\text { M. and } \\
\text { Mann D. G. }\end{array}$ & 1 & - & 1 & 1 & 1 & 1 & $\infty$ & 1 & है & 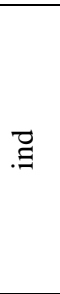 & ᄅ & $\mathscr{Z}$ & $\stackrel{L}{0}$ & o & $\frac{\mathscr{v}}{\tilde{v}}$ \\
\hline 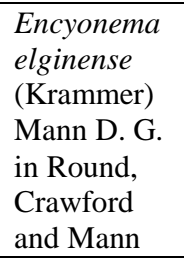 & 1 & $\neg$ & $r$ & 1 & 1 & 1 & $\infty$ & 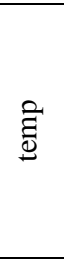 & $\vec{\omega}$ & 㫕 & ᄅ & $\stackrel{x}{n}$ & $\stackrel{\text { Lִ }}{\rightarrow}$ & 1 & 1 \\
\hline $\begin{array}{l}\text { Encyonema } \\
\text { pergracile } \\
\text { Krammer }\end{array}$ & 1 & $\neg$ & - & 1 & 1 & -1 & $\infty$ & 1 & 1 & 1 & 1 & 1 & 1 & 1 & 1 \\
\hline $\begin{array}{l}\text { Encyonema } \\
\text { prostratum } \\
\text { (Berkeley) } \\
\text { Kützing }\end{array}$ & 1 & $\neg$ & 1 & 1 & 1 & 1 & $\stackrel{m}{a}$ & 1 & 竞 & चै & $-r$ & $\mathscr{\Xi}$ & $\stackrel{m}{\rightarrow}$ & 0 & $\frac{\mathscr{d}}{\tilde{U}}$ \\
\hline $\begin{array}{l}\text { Epithemia } \\
\text { adnata } \\
\text { (Kützing) } \\
\text { Brébisson }\end{array}$ & -1 & $\neg$ & 1 & 1 & 1 & 1 & $\infty$ & 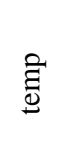 & $\vec{\omega}$ & $\vec{\sigma}$ & $-r$ & $\stackrel{x}{\infty}$ & $\stackrel{\sim}{-}$ & $\stackrel{\Xi}{\Xi}$ & $\frac{0}{\pi}$ \\
\hline
\end{tabular}


Table 2 (continued): Diversity and ecology of diatom algae in the thermal and mineral water sources of Pamir with species autecology. Number of springs 1-6 as in table 1.

\begin{tabular}{|c|c|c|c|c|c|c|c|c|c|c|c|c|c|c|c|}
\hline Таха & -1 & $\sim$ & $m$ & $\nabla$ & เ & 0 & त्ञ & $H$ & $\overrightarrow{\hat{a}}$ & 竞 & 茎 & D & స్心ే & $\stackrel{O}{\Theta}$ & 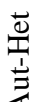 \\
\hline $\begin{array}{l}\text { Epithemia } \\
\text { adnata var. } \\
\text { porcellus } \\
\text { (Kützing) } \\
\text { Ross R. }\end{array}$ & -1 & | & 1 & 1 & 1 & 1 & 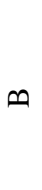 & 1 & 1 & 㫕 & $\cdot-1$ & 1 & $\stackrel{\circ}{\mathrm{i}}$ & $\stackrel{\Xi}{\Xi}$ & 1 \\
\hline $\begin{array}{l}\text { Epithemia } \\
\text { adnata var. } \\
\text { saxonica } \\
\text { (Kützing) } \\
\text { Patrick R. M. } \\
\text { in Patrick } \\
\text { and Reimer }\end{array}$ & | & I & -1 & 1 & 1 & 1 & 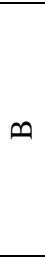 & 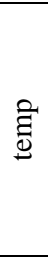 & 1 & 㫕 & $\cdot \rightarrow$ & I & ִָ & $\stackrel{\mathscr{\Xi}}{\Xi}$ & 1 \\
\hline $\begin{array}{l}\text { Epithemia } \\
\text { argus } \\
\text { (Ehrenberg) } \\
\text { Kützing }\end{array}$ & -1 & | & | & 1 & 1 & 1 & $\stackrel{n}{\stackrel{1}{a}}$ & 1 & $\begin{array}{l}5 \\
\frac{1}{1} \\
\frac{1}{\omega}\end{array}$ &.$\Xi$ & $\rightarrow r$ & $\mathscr{c}$ & $\hat{o}$ & $\Xi$ & 1 \\
\hline $\begin{array}{l}\text { Epithemia } \\
\text { argus var. } \\
\text { angusta } \\
\text { Tarnavschi }\end{array}$ & | & I & $\neg$ & 1 & 1 & 1 & 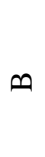 & 1 & 1 &.$\Xi$ & $\cdot \rightarrow$ & 1 & 1 & 1 & 1 \\
\hline $\begin{array}{l}\text { Epithemia } \\
\text { argus var. } \\
\text { longicornis } \\
\text { (Ehrenberg) } \\
\text { Grunow }\end{array}$ & -1 & I & | & I & 1 & 1 & $\stackrel{m}{1}$ & 1 & 1 &.$\Xi$ & $\rightarrow$ & 1 & 1 & 1 & I \\
\hline $\begin{array}{l}\text { Epithemia } \\
\text { sorex } \\
\text { Kützing }\end{array}$ & | & -1 & 1 & I & 1 & -1 & 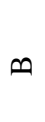 & 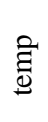 & $\begin{array}{l}5 \\
\text { 点 } \\
\frac{1}{n}\end{array}$ & $\frac{\Psi}{\pi}$ & $\rightarrow$ & $\stackrel{x}{n}$ & تُ & 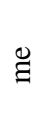 & $\frac{\tilde{U}}{\pi}$ \\
\hline $\begin{array}{l}\text { Epithemia } \\
\text { turgida } \\
\text { (Ehrenberg) } \\
\text { Kützing }\end{array}$ & | & | & $\neg$ & 1 & 1 & 1 & $ص$ & 官 & $\vec{\omega}$ & तี & $\rightarrow$ & $\stackrel{x}{\omega}$ & O̊ & 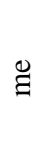 & $\frac{n}{\pi}$ \\
\hline $\begin{array}{l}\text { Epithemia } \\
\text { turgida var. } \\
\text { capitata } \\
\text { Fricke }\end{array}$ & -1 & | & I & I & 1 & 1 & 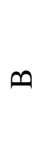 & 1 & 1 & त्व & $\rightarrow$ & 1 & 1 & 1 & I \\
\hline
\end{tabular}


Table 2 (continued): Diversity and ecology of diatom algae in the thermal and mineral water sources of Pamir with species autecology. Number of springs 1-6 as in table 1.

\begin{tabular}{|c|c|c|c|c|c|c|c|c|c|c|c|c|c|c|c|}
\hline Таха & -1 & $N$ & $m$ & $\nabla$ & L & 0 & 苂 & $\mapsto$ & 㐫 & 宫 & 苂 & D & దే & $\stackrel{8}{日}$ & 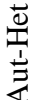 \\
\hline $\begin{array}{l}\text { Epithemia } \\
\text { turgida var. } \\
\text { granulata } \\
\text { (Ehrenberg) } \\
\text { Brun }\end{array}$ & 1 & 1 & 1 & 1 & 1 & $\dashv$ & $\infty$ & 1 & $\ddot{\omega}$ & $\frac{\omega}{\pi}$ & $-r$ & 1 & g. & ఫ్ & 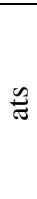 \\
\hline $\begin{array}{l}\text { Eucocconeis } \\
\text { flexella } \\
\text { (Kützing) } \\
\text { Meister }\end{array}$ & - & 1 & 1 & I & 1 & 1 & $\infty$ & 1 & है &.$\Xi$ & 夆 & 的 & กี & $\ddot{o}$ & $\frac{\mathscr{v}}{\pi}$ \\
\hline $\begin{array}{l}\text { Eunotia } \\
\text { diodon } \\
\text { Ehrenberg }\end{array}$ & - & 1 & 1 & I & 1 & 1 & $\infty$ & 8 & $\ddot{\omega}$ & $\widetilde{\widetilde{U}}$ & $-r$ & 1 & $\tilde{o}$ & t & $\frac{\mathscr{m}}{\pi}$ \\
\hline $\begin{array}{l}\text { Eunotia } \\
\text { faba } \\
\text { Ehrenberg }\end{array}$ & 1 & 1 & 1 & -1 & 1 & 1 & $\infty$ & 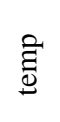 & $\begin{array}{l}\frac{5}{4} \\
\frac{1}{\omega}\end{array}$ & 㫕 & $-r$ & $\stackrel{x}{\infty}$ & $\stackrel{-}{ت}$ & ఫ్ & $\frac{n}{\pi}$ \\
\hline $\begin{array}{l}\text { Fragilaria } \\
\text { capucina var. } \\
\text { vaucheriae } \\
\text { (Kützing) } \\
\text { Lange- } \\
\text { Bertalot } \\
\end{array}$ & 1 & -1 & 1 & I & 1 & 1 & $\begin{array}{l}\text { ज्ञ } \\
\text { की } \\
\text { â }\end{array}$ & 1 & $\begin{array}{l}\frac{t}{\omega} \\
\frac{1}{\omega}\end{array}$ & 㫕 & $r$ & 的 & 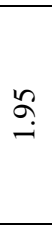 & 0 & $\frac{\mathscr{g}}{\pi}$ \\
\hline $\begin{array}{l}\text { Fragilaria } \\
\text { tenera } \\
\text { (Smith W.) } \\
\text { Lange- } \\
\text { Bertalot }\end{array}$ & $\neg$ & 1 & 1 & I & 1 & 1 & $\stackrel{m}{a}$ & 1 & ह & $\underset{\tau}{\tilde{U}}$ & ᄅ & $\stackrel{x}{n}$ & $\stackrel{n}{i}$ & ఫ్ & $\frac{\mathscr{n}}{\tilde{\sigma}}$ \\
\hline $\begin{array}{l}\text { Fragilarifor- } \\
\text { ma virescens } \\
\text { (Ralfs) } \\
\text { Williams D. } \\
\text { M. and Round }\end{array}$ & $\neg$ & 1 & 1 & I & 1 & 1 & $\stackrel{m}{a}$ & 1 & $\ddot{\omega}$ & $\overrightarrow{.}$ & $r$ & $\approx$ & $\stackrel{\nabla}{0}$ & है & $\frac{\mathscr{v}}{\pi}$ \\
\hline 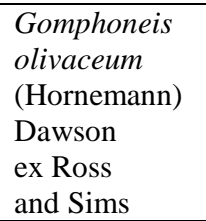 & - & 1 & 1 & I & 1 & 1 & $\infty$ & | & $\begin{array}{l}\frac{\bar{v}}{p} \\
\frac{1}{\omega}\end{array}$ & 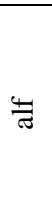 & $\rightarrow$ & $\approx$ & $\stackrel{\text { 导 }}{-}$ & 0 & $\frac{\mathscr{v}}{\tilde{\tau}}$ \\
\hline
\end{tabular}


Table 2 (continued): Diversity and ecology of diatom algae in the thermal and mineral water sources of Pamir with species autecology. Number of springs 1-6 as in table 1.

\begin{tabular}{|c|c|c|c|c|c|c|c|c|c|c|c|c|c|c|c|}
\hline Таха & -1 & $\sim$ & $m$ & $\nabla$ & $\llcorner 0$ & 0 & 苂 & H & ô & I & 苂 & D & 触 & $\stackrel{g}{\Theta}$ & 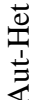 \\
\hline $\begin{array}{l}\text { Gomphonema } \\
\text { angustatum } \\
\text { (Kützing) } \\
\text { Rabenhorst }\end{array}$ & 1 & -1 & 1 & 1 & 1 & 1 & $\infty$ & 1 & $\begin{array}{l}\frac{\vec{n}}{4} \\
\frac{1}{\omega}\end{array}$ &.$\Xi$ & $-r$ & 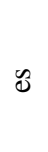 & $\stackrel{m}{-}$ & 芳 & 1 \\
\hline $\begin{array}{l}\text { Gomphonema } \\
\text { longiceps } \\
\text { Ehrenberg }\end{array}$ & 1 & $\neg$ & 1 & 1 & - & 1 & $\infty$ & 1 & ह &.$\Xi$ & $r$ & $\mathscr{E}$ & $\stackrel{\nabla}{0}$ & 1 & 1 \\
\hline $\begin{array}{l}\text { Gomphonema } \\
\text { longiceps var. } \\
\text { subclavatum } \\
\text { Grunow in } \\
\text { Schneider }\end{array}$ & 1 & - & 1 & 1 & 1 & 1 & $\infty$ & 1 & है & $\Xi$ & $-r$ & 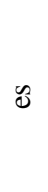 & $\stackrel{\leftrightarrow}{-}$ & 1 & 1 \\
\hline $\begin{array}{l}\text { Gomphonema } \\
\text { productum } \\
\text { (Grunow) } \\
\text { Lange- } \\
\text { Bertalot and } \\
\text { Reichardt } \\
\text { in Lange- } \\
\text { Bertalot }\end{array}$ & - & 1 & 1 & 1 & 1 & 1 & $\infty$ & 1 & 音 &.$\Xi$ & $-r$ & $\approx$ & $\stackrel{m}{-}$ & 芳 & $\stackrel{\mathbb{\sigma}}{*}$ \\
\hline $\begin{array}{l}\text { Gomphonema } \\
\text { ventricosum } \\
\text { Gregory }\end{array}$ & 1 & - & 1 & 1 & 1 & 1 & $\infty$ & $\overline{8}$ & क & 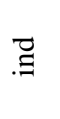 & $-r$ & 1 & חִ & t & $\frac{\mathscr{U}}{\tilde{\sigma}}$ \\
\hline $\begin{array}{l}\text { Gomphonema } \\
\text { gracile } \\
\text { Ehrenberg }\end{array}$ & - & 1 & 1 & 1 & 1 & - & 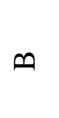 & 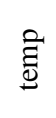 & $\vec{\omega}$ & 㫕 & $-r$ & 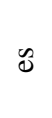 & $\stackrel{\infty}{0}$ & $\Xi$ & $\frac{0}{\pi}$ \\
\hline $\begin{array}{l}\text { Halamphora } \\
\text { acutiuscula } \\
\text { (Kützing) } \\
\text { Levkov }\end{array}$ & I & -1 & 1 & 1 & 1 & 1 & $\stackrel{m}{a}$ & $\begin{array}{l}E \\
\text { E } \\
3\end{array}$ & 1 & 㫕 & 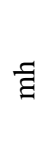 & के & $\stackrel{\circ}{i}$ & 1 & 1 \\
\hline $\begin{array}{l}\text { Halamphora } \\
\text { coffeaeformis } \\
\text { (Agardh) } \\
\text { Levkov }\end{array}$ & 1 & 1 & 1 & 1 & - & 1 & $\infty$ & 1 & $\begin{array}{l}\vec{w} \\
\frac{\vec{n}}{\omega}\end{array}$ & $\frac{\Psi}{\sigma}$ & 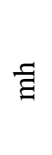 & 1 & $\stackrel{0}{\dot{m}}$ & 0 & $\frac{\mathscr{v}}{\pi}$ \\
\hline
\end{tabular}


Table 2 (continued): Diversity and ecology of diatom algae in the thermal and mineral water sources of Pamir with species autecology. Number of springs 1-6 as in table 1.

\begin{tabular}{|c|c|c|c|c|c|c|c|c|c|c|c|c|c|c|c|}
\hline Таха & -1 & $N$ & $m$ & $\nabla$ & เก & 0 & 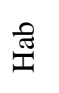 & $H$ & ô & I & 莡 & D & जि & $\stackrel{8}{9}$ & 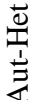 \\
\hline $\begin{array}{l}\text { Halamphora } \\
\text { subcapitata } \\
\text { (Kisselew) } \\
\text { Levkov }\end{array}$ & 1 & 1 & -1 & 1 & 1 & 1 & $\infty$ & 1 & 1 & 1 & $\exists$ & 1 & 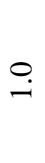 & 1 & 1 \\
\hline $\begin{array}{l}\text { Halamphora } \\
\text { veneta } \\
\text { (Kützing) } \\
\text { Levkov }\end{array}$ & - & 1 & 1 & 1 & 1 & 1 & $\infty$ & 1 & $\begin{array}{l}\frac{v}{0} \\
\dot{1} \\
\omega\end{array}$ & $\frac{\Psi}{\tau}$ & $-r$ & ש & 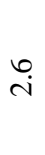 & 0 & $\frac{\mathscr{v}}{\pi}$ \\
\hline 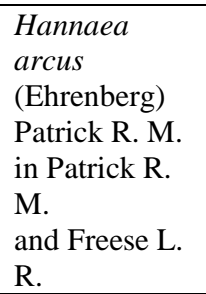 & - & -1 & -1 & 1 & 1 & 1 & $\infty$ & छ్ & है & 㫕 & $-r$ & 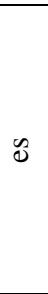 & חֶ. & ఫ̄ & $\frac{\mathscr{n}}{\tilde{v}}$ \\
\hline $\begin{array}{l}\text { Humidophila } \\
\text { perpusilla } \\
\text { (Grunow) } \\
\text { Lowe at al. }\end{array}$ & -1 & 1 & 1 & 1 & 1 & 1 & $\infty$ & 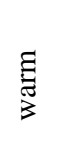 & 紊 &. $\bar{g}$ & $r$ & के & $\hat{o}$ & ह̦ & $\frac{\mathscr{m}}{\pi}$ \\
\hline $\begin{array}{l}\text { Kurtkrammeri } \\
\text { a aequalis } \\
\text { (Smith W.) } \\
\text { Bahls L. }\end{array}$ & -1 & 1 & 1 & 1 & 1 & 1 & 竞 & 1 & 1 & 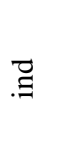 & $-r$ & 1 & $\stackrel{\leftrightarrow}{\circ}$ & t & 1 \\
\hline $\begin{array}{l}\text { Mastogloia } \\
\text { smithii } \\
\text { Thwaites }\end{array}$ & -1 & 1 & 1 & 1 & 1 & 1 & $\infty$ & 1 & 1 & 㫕 & है & $\stackrel{x}{n}$ & $\stackrel{m}{-}$ & $\ddot{\Xi}$ & 1 \\
\hline $\begin{array}{l}\text { Meridion } \\
\text { circulare } \\
\text { (Greville) } \\
\text { Agardh }\end{array}$ & - & 1 & 1 & 1 & 1 & 1 & $\infty$ & 1 & ह &.$\Xi$ & $-r$ & ¿ & $ت$ & 兑 & $\frac{\mathscr{g}}{\pi}$ \\
\hline $\begin{array}{l}\text { Meridion } \\
\text { circulare var. } \\
\text { constrictum } \\
\text { (Ralfs) Brun }\end{array}$ & -1 & 1 & 1 & 1 & 1 & 1 & $\stackrel{m}{a}$ & 1 & $\begin{array}{l}\vec{w} \\
\frac{\vec{n}}{n}\end{array}$ & 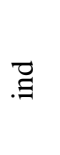 & 르 & $\underset{n}{x}$ & 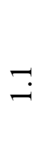 & $\begin{array}{l}u \\
\vdots\end{array}$ & $\frac{\mathscr{v}}{\pi}$ \\
\hline
\end{tabular}


Table 2 (continued): Diversity and ecology of diatom algae in the thermal and mineral water sources of Pamir with species autecology. Number of springs 1-6 as in table 1.

\begin{tabular}{|c|c|c|c|c|c|c|c|c|c|c|c|c|c|c|c|}
\hline Таха & -1 & $\sim$ & $m$ & $\nabla$ & L & 0 & 苂 & $H$ & 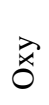 & 兑 & 胥 & D & స్టి & 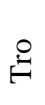 & 营 \\
\hline $\begin{array}{l}\text { Meridion } \\
\text { lineare } \\
\text { Williams }\end{array}$ & -1 & -1 & 1 & - & I & 1 & 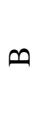 & 1 & 离 & . & $-r$ & $\approx$ & ت̆ & ह̦ & ثี兀 \\
\hline $\begin{array}{l}\text { Navicula } \\
\text { brasiliensis } \\
\text { Grunow }\end{array}$ & | & -1 & 1 & 1 & 1 & 1 & 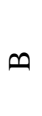 & 1 & I & 1 & 1 & 1 & 1 & 1 & 1 \\
\hline $\begin{array}{l}\text { Navicula } \\
\text { cincta } \\
\text { (Ehrenberg) } \\
\text { Ralfs }\end{array}$ & -1 & I & 1 & 1 & 1 & 1 & $ص$ & 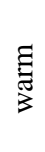 & $\begin{array}{l}\frac{1}{5} \\
\frac{1}{\omega}\end{array}$ & 㫕 & $I$ & $\mathscr{Q}$ & ᄂ? & 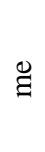 & تँّ \\
\hline $\begin{array}{l}\text { Navicula } \\
\text { cryptocephala } \\
\text { Kützing }\end{array}$ & -1 & 1 & 1 & 1 & 1 & 1 & $\frac{m}{\rho^{\prime}}$ & 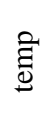 & $\begin{array}{l}\frac{1}{4} \\
\frac{1}{\omega}\end{array}$ &.$\Xi$ & $-r$ & $\mathscr{E}$ & $\breve{i}$ & İ & ثี兀 \\
\hline $\begin{array}{l}\text { Navicula } \\
\text { cryptocephala } \\
\text { var. lata } \\
\text { Poretzky and } \\
\text { Anisimova }\end{array}$ & -1 & 1 & 1 & 1 & 1 & 1 & 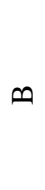 & 1 & 1 & 1 & $\neg$ & 1 & 1 & 1 & 1 \\
\hline $\begin{array}{l}\text { Navicula } \\
\text { dicephala } \\
\text { Ehrenberg }\end{array}$ & - & 1 & 1 & 1 & 1 & 1 & 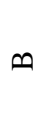 & 1 & 1 &.$\Xi$ & $r$ & 1 & $\stackrel{+}{\sim}$ & 1 & 1 \\
\hline $\begin{array}{l}\text { Navicula } \\
\text { digitoradiata } \\
\text { (Gregory) } \\
\text { Ralfs in } \\
\text { Prichard }\end{array}$ & 1 & 1 & 1 & - & 1 & 1 & $ص$ & 1 & 1 & $\frac{\omega}{\pi}$ & - & $\mathscr{\theta}$ & $\stackrel{\circ}{i}$ & $\stackrel{\mathscr{\Xi}}{\Xi}$ & 1 \\
\hline $\begin{array}{l}\text { Navicula } \\
\text { gothlandica } \\
\text { Grunow }\end{array}$ & -1 & 1 & 1 & 1 & 1 & 1 & $\stackrel{n}{1}$ & 1 & I & $\frac{\bar{\pi}}{\pi}$ & $I$ & $\mathscr{\theta}$ & $\stackrel{\circ}{i}$ & క్ & 1 \\
\hline $\begin{array}{l}\text { Navicula } \\
\text { lacustris var. } \\
\text { paulseniana } \\
\text { (Petersen J. } \\
\text { B.) Zabelina }\end{array}$ & 1 & 1 & 1 & 1 & 1 & -1 & 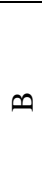 & 1 & 1 & 1 & 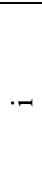 & 1 & 1 & 1 & 1 \\
\hline
\end{tabular}


Table 2 (continued): Diversity and ecology of diatom algae in the thermal and mineral water sources of Pamir with species autecology. Number of springs 1-6 as in table 1.

\begin{tabular}{|c|c|c|c|c|c|c|c|c|c|c|c|c|c|c|c|}
\hline Taxa & - & $\sim$ & $m$ & $\nabla$ & م & 0 & 茎 & 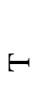 & ஷ্் & 䄍 & 茎 & D & స్心 & $\stackrel{ }{\varrho}$ & 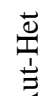 \\
\hline $\begin{array}{l}\text { Navicula } \\
\text { lanceolata } \\
\text { (Agardh) } \\
\text { Ehrenberg }\end{array}$ & 1 & $\neg$ & 1 & 1 & 1 & 1 & 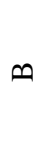 & 1 & $\begin{array}{l}5 \\
\overline{1} \\
\frac{1}{\omega}\end{array}$ & 㫕 & 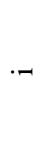 & $\approx$ & $\stackrel{L}{N}$ & 0 & 党 \\
\hline $\begin{array}{l}\text { Navicula } \\
\text { lucidula } \\
\text { Grunow }\end{array}$ & 1 & 1 & 1 & 1 & 1 & -1 & $ص$ & 1 & 1 & 1 & $\rightarrow-1$ & 1 & 1 & 1 & 1 \\
\hline $\begin{array}{l}\text { Navicula } \\
\text { peregrina } \\
\text { (Ehrenberg) } \\
\text { Kützing }\end{array}$ & 1 & 1 & 1 & -1 & 1 & 1 & $\stackrel{\infty}{1}$ & 1 & I & $\frac{\Psi}{\pi}$ & हี & $\mathscr{Q}$ & I? & $\begin{array}{l}\Xi \\
\vdots \\
0\end{array}$ & 1 \\
\hline $\begin{array}{l}\text { Navicula } \\
\text { radiosa var. } \\
\text { tenella } \\
\text { (Brébisson) } \\
\text { Cleve and } \\
\text { Möller }\end{array}$ & 1 & 1 & 1 & -1 & 1 & 1 & $\stackrel{m}{\stackrel{1}{a}}$ & 1 & 1 & 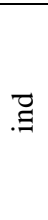 & $\rightarrow$ & $\stackrel{x}{\omega}$ & $\stackrel{m}{-}$ & ఫ̦ & 1 \\
\hline $\begin{array}{l}\text { Navicula } \\
\text { rhynchoce- } \\
\text { phala } \\
\text { Ehrenberg }\end{array}$ & 1 & -1 & 1 & 1 & 1 & 1 & $ص$ & 1 & I & 㫕 & $I$ & 1 & 亗 & E̦ & 䒕 \\
\hline $\begin{array}{l}\text { Navicula } \\
\text { rostellata } \\
\text { Kützing }\end{array}$ & 1 & 1 & 1 & 1 & 1 & -1 & $ص$ & 1 & $\begin{array}{l}\frac{n}{4} \\
\frac{1}{\omega}\end{array}$ & $\frac{W}{\pi}$ & $\cdot-1$ & ש & $\underset{\sim}{\sim}$ & 0 & ثै \\
\hline $\begin{array}{l}\text { Navicula } \\
\text { rotaeana } \\
\text { (Rabenhorst) } \\
\text { Grunow }\end{array}$ & 1 & -1 & 1 & 1 & 1 & 1 & 皇 & 1 & $\vec{\omega}$ & $\vec{\Xi}$ & $\rightarrow-1$ & 1 & $\hat{o}$ & $\tilde{\sigma}$ & 1 \\
\hline $\begin{array}{l}\text { Navicula } \\
\text { scutum } \\
\text { Schumann }\end{array}$ & 1 & 1 & 1 & 1 & -1 & 1 & $ص$ & 1 & 1 & I & I & 1 & I & 1 & 1 \\
\hline
\end{tabular}


Table 2 (continued): Diversity and ecology of diatom algae in the thermal and mineral water sources of Pamir with species autecology. Number of springs 1-6 as in table 1.

\begin{tabular}{|c|c|c|c|c|c|c|c|c|c|c|c|c|c|c|c|}
\hline Таха & -1 & $\sim$ & $m$ & $\nabla$ & L & 0 & 苂 & $H$ & ồ & 焉 & 胥 & D & స్ & 㫣 & 苞 \\
\hline $\begin{array}{l}\text { Navicula } \\
\text { tripunctata } \\
\text { (Müller O. F.) } \\
\text { Bory in Bory } \\
\text { de Saint- } \\
\text { Vincent }\end{array}$ & 1 & -1 & 1 & 1 & 1 & 1 & $\stackrel{n}{n}$ & I & $\begin{array}{l}5 \\
\frac{1}{\omega} \\
\frac{1}{\omega}\end{array}$ &.$\Xi$ & $r$ & $\mathscr{0}$ & $\hat{-}$ & 0 & $\frac{\ddot{\sigma}}{\pi}$ \\
\hline $\begin{array}{l}\text { Navicula } \\
\text { tuscula f. } \\
\text { obtusa } \\
\text { (Hustedt) } \\
\text { Proschkina- } \\
\text { Lavrenko } \\
\end{array}$ & 1 & 1 & 1 & I & 1 & -1 & $\frac{m}{n^{\prime}}$ & 1 & 1 & $\frac{\mathbb{\sigma}}{\sigma}$ & $\neg$ & 1 & $\stackrel{\text { Nִ }}{-1}$ & 志 & 1 \\
\hline $\begin{array}{l}\text { Navicula } \\
\text { viridula } \\
\text { (Kützing) } \\
\text { Ehrenberg }\end{array}$ & 1 & 1 & $\neg$ & 1 & 1 & 1 & 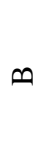 & 1 & $\begin{array}{l}5 \\
\bar{n} \\
\frac{1}{\omega}\end{array}$ & $\stackrel{\Psi}{\sigma}$ & $I$ & $\mathscr{c}$ & $\stackrel{\sim}{N}$ & $\stackrel{\Xi}{\Xi}$ & $\stackrel{\nabla}{\pi}$ \\
\hline $\begin{array}{l}\text { Neidiomorpha } \\
\text { binodis } \\
\text { (Ehrenberg) } \\
\text { Cantonati M., } \\
\text { Lange- } \\
\text { Bertalot and } \\
\text { Angeli N. }\end{array}$ & $\neg$ & 1 & 1 & 1 & 1 & 1 & 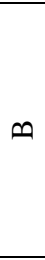 & 1 & 离 & $\bar{\tau}$ & $\neg$ & 1 & نِ & 气 & تِّ \\
\hline $\begin{array}{l}\text { Neidium } \\
\text { Affine } \\
\text { (Ehrenberg) } \\
\text { Pfizer }\end{array}$ & 1 & $\neg$ & 1 & 1 & 1 & 1 & 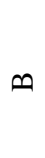 & 1 & 离 &.$\Xi$ & $-r$ & 1 & $\hat{0}$ & $\tilde{o}$ & 䒕 \\
\hline $\begin{array}{l}\text { Neidium } \\
\text { affine var. } \\
\text { undulatum } \\
\text { (Grunow) } \\
\text { Cleve }\end{array}$ & 1 & -1 & 1 & I & 1 & 1 & 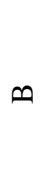 & 1 & 离 &.$\Xi$ & $r$ & 1 & $\hat{0}$ & $\overrightarrow{0}$ & 䒕 \\
\hline $\begin{array}{l}\text { Neidium } \\
\text { productum } \\
\text { (Smith W.) } \\
\text { Cleve }\end{array}$ & 1 & -1 & 1 & 1 & 1 & 1 & $\stackrel{m}{\rho_{1}^{\prime}}$ & 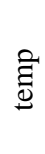 & 1 & . & $\rightarrow$ & $\stackrel{x}{\infty}$ & $\ddot{0}$ & ठ & $\frac{n}{\pi}$ \\
\hline $\begin{array}{l}\text { Nitzschia } \\
\text { amphibian } \\
\text { Grunow }\end{array}$ & -1 & 1 & 1 & 1 & 1 & 1 & $\begin{array}{l}n \\
n^{\prime} \\
n^{\prime}\end{array}$ & 䓌 & $\begin{array}{l}\frac{n}{\omega} \\
\frac{1}{w}\end{array}$ & $\bar{\tau}$ & $\rightarrow$ & के & $\vec{i}$ & 0 & 三 \\
\hline
\end{tabular}


Table 2 (continued): Diversity and ecology of diatom algae in the thermal and mineral water sources of Pamir with species autecology. Number of springs 1-6 as in table 1.

\begin{tabular}{|c|c|c|c|c|c|c|c|c|c|c|c|c|c|c|c|}
\hline Таха & -1 & $N$ & $m$ & $\nabla$ & L & 0 & $\vec{\pi}$ & $H$ & ồ & II & 苂 & D & ڤే & 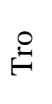 & $\begin{array}{l}\stackrel{\vec{T}}{1} \\
\end{array}$ \\
\hline $\begin{array}{l}\text { Nitzschia } \\
\text { amphibia var. } \\
\text { thermalis } \\
\text { Grunow }\end{array}$ & -1 & 1 & 1 & 1 & 1 & 1 & $\emptyset$ & 1 & 1 & 1 & 1 & 1 & 1 & 1 & 1 \\
\hline $\begin{array}{l}\text { Nitzschia } \\
\text { dissipata } \\
\text { (Kützing) } \\
\text { Rabenhorst }\end{array}$ & -1 & 1 & 1 & 1 & 1 & 1 & $\infty$ & 1 & $\begin{array}{l}\frac{\omega}{5} \\
\frac{1}{\omega}\end{array}$ & సै & -7 & 秥 & $\stackrel{\text { I }}{-}$ & 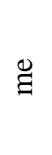 & 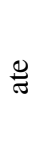 \\
\hline $\begin{array}{l}\text { Nitzschia } \\
\text { dubia } \\
\text { Smith W. }\end{array}$ & -1 & 1 & 1 & 1 & 1 & 1 & $\stackrel{m}{1}$ & 1 & $\begin{array}{l}\frac{v}{5} \\
\frac{1}{\omega}\end{array}$ & गै & -7 & 1 & $\widehat{i}$ & 0 & Ӗ \\
\hline $\begin{array}{l}\text { Nitzschia } \\
\text { fasciculate } \\
\text { (Grunow) } \\
\text { Grunow } \\
\text { in Van Heurck }\end{array}$ & | & 1 & 1 & - & 1 & 1 & $m$ & 1 & $\ddot{\omega}$ & సै & $I$ & $\stackrel{x}{n}$ & $ت$ & 1 & 1 \\
\hline $\begin{array}{l}\text { Nitzschia } \\
\text { gracilis } \\
\text { Hantzsch }\end{array}$ & -1 & 1 & 1 & I & 1 & 1 & $\stackrel{m}{\prime \prime}$ & 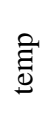 & $\begin{array}{l}\frac{\bar{p}}{5} \\
\frac{1}{\omega}\end{array}$ & $\vec{\Xi}$ &. & के & . & $\Xi$ & 1 \\
\hline $\begin{array}{l}\text { Nitzschia } \\
\text { gradifera } \\
\text { Hustedt }\end{array}$ & | & I & 1 & I & -1 & 1 & $\infty$ & 1 & 1 & 1 & $I$ & 1 & 1 & 1 & 1 \\
\hline $\begin{array}{l}\text { Nitzschia } \\
\text { linearis } \\
\text { (Agardh) } \\
\text { Smith W. }\end{array}$ & -1 & 1 & 1 & I & 1 & 1 & $\infty$ & 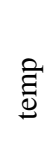 & $\begin{array}{l}\frac{1}{5} \\
\frac{1}{\omega n}\end{array}$ & సै & -7 & 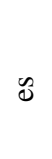 & Fi & छั & $\frac{\mathscr{0}}{\pi}$ \\
\hline $\begin{array}{l}\text { Nitzschia } \\
\text { linearis var. } \\
\text { tenuis } \\
\text { (Smith W.) } \\
\text { Grunow in } \\
\text { Cleve and } \\
\text { Grunow }\end{array}$ & 1 & 1 & 1 & I & 1 & -1 & $\infty$ & 1 & ह & సี & $\rightarrow$ & $\approx$ & fi & 气 & 1 \\
\hline
\end{tabular}


Table 2 (continued): Diversity and ecology of diatom algae in the thermal and mineral water sources of Pamir with species autecology. Number of springs 1-6 as in table 1.

\begin{tabular}{|c|c|c|c|c|c|c|c|c|c|c|c|c|c|c|c|}
\hline Таха & -1 & $N$ & $m$ & $\nabla$ & L & 0 & 苂 & $H$ & ঝे & $\frac{I}{2}$ & 蒫 & D & స్心ే & ڤ & $\begin{array}{l}\stackrel{\vec{c}}{T} \\
\stackrel{ \pm}{\Xi}\end{array}$ \\
\hline $\begin{array}{l}\text { Nitzschia } \\
\text { sublinearis } \\
\text { Hustedt }\end{array}$ & | & -1 & 1 & I & 1 & 1 & $\stackrel{m}{a}^{m}$ & 1 & 1 & 㫕 & -1 & 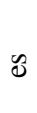 & $\stackrel{\circ}{\text { ले }}$ & $\stackrel{\mathscr{E}}{ }$ & I \\
\hline $\begin{array}{l}\text { Nitzschia } \\
\text { subtilis } \\
\text { (Kützing) } \\
\text { Grunow } \\
\text { in Cleve and } \\
\text { Grunow }\end{array}$ & | & -1 & 1 & I & 1 & I & $D$ & 1 & 1 & $\frac{\Psi}{\pi}$ & $\neg$ & 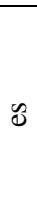 & $\stackrel{\overbrace{}}{-}$ & 竞 & 1 \\
\hline $\begin{array}{l}\text { Nitzschia } \\
\text { thermalis } \\
\text { (Ehrenberg) } \\
\text { Auerswald in } \\
\text { Rabenhorst }\end{array}$ & -1 & 1 & 1 & I & 1 & I & D & 1 & 1 & ?. & $\neg$ & $\mathscr{\theta}$ & $\stackrel{\infty}{\sim}$ & 1 & I \\
\hline $\begin{array}{l}\text { Nitzschia } \\
\text { thermalis } \\
\text { var. minor } \\
\text { Hilse }\end{array}$ & -1 & 1 & 1 & I & 1 & I & 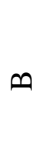 & 1 & $\begin{array}{l}\frac{n}{4} \\
\frac{1}{\omega}\end{array}$ & 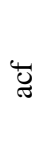 & 1 & 1 & نـ & I & I \\
\hline $\begin{array}{l}\text { Nitzschia } \\
\text { vermicularis } \\
\text { (Kützing) } \\
\text { Hantzsch in } \\
\text { Rabenhorst }\end{array}$ & 1 & 1 & -1 & I & 1 & I & $\stackrel{m}{n}$ & 1 & 紊 & 䒕 &. & 1 & ָָ & $\Xi$ & I \\
\hline $\begin{array}{l}\text { Parlibellus } \\
\text { crucicula } \\
\text { (Smith W.) } \\
\text { Witkowski, } \\
\text { Lange- } \\
\text { Bertalot and } \\
\text { Metzeltin } \\
\end{array}$ & -1 & 1 & 1 & I & 1 & I & 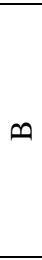 & 1 & 1 &.$\Xi$ & $\bar{\Xi}$ & ' & $\stackrel{\circ}{N}$ & 1 & I \\
\hline $\begin{array}{l}\text { Pinnularia } \\
\text { appendiculata } \\
\text { (Agardh C.) } \\
\text { Schaarschmidt }\end{array}$ & -1 & 1 & I & I & I & I & $D$ & 1 & 竞 &. &. & $\mathscr{d}$ & $\stackrel{n}{0}$ & ఫ్ & $\frac{\tilde{v}}{\tilde{\tau}}$ \\
\hline $\begin{array}{l}\text { Pinnularia } \\
\text { elegans } \\
\text { (Smith W.) } \\
\text { Krammer K. }\end{array}$ & -1 & 1 & 1 & I & 1 & I & 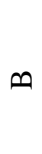 & 1 & 1 & $\frac{\omega}{\pi}$ & I & 1 & $\stackrel{\circ}{\sim}$ & $\Xi$ & 1 \\
\hline
\end{tabular}


Table 2 (continued): Diversity and ecology of diatom algae in the thermal and mineral water sources of Pamir with species autecology. Number of springs 1-6 as in table 1.

\begin{tabular}{|c|c|c|c|c|c|c|c|c|c|c|c|c|c|c|c|}
\hline Таха & -1 & $N$ & $m$ & $\nabla$ & เn & 0 & ত్రి & $H$ & ô & 蛋 & 苂 & D & लే & $\stackrel{8}{9}$ & 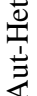 \\
\hline $\begin{array}{l}\text { Pinnularia } \\
\text { fonticola } \\
\text { Hustedt }\end{array}$ & - & 1 & 1 & 1 & 1 & 1 & 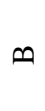 & 1 & 1 &.$\Xi$ & $-r$ & 1 & 1 & 1 & 1 \\
\hline $\begin{array}{l}\text { Pinnularia } \\
\text { gibbiformis } \\
\text { Krammer K. }\end{array}$ & -1 & 1 & 1 & 1 & 1 & 1 & $\infty$ & 1 & 1 & 1 & 1 & 1 & $\dddot{m}$ & 1 & 1 \\
\hline $\begin{array}{l}\text { Pinnularia } \\
\text { lata } \\
\text { (Brébisson) } \\
\text { Smith W. }\end{array}$ & -1 & 1 & 1 & 1 & 1 & 1 & $a_{a}^{m}$ & 1 & है & $\mathbb{Z}_{\tilde{\tau}}$ & $-r$ & 1 & $\stackrel{\circ}{\rightarrow}$ & t & 1 \\
\hline $\begin{array}{l}\text { Pinnularia } \\
\text { microstauron } \\
\text { (Ehrenberg) } \\
\text { Cleve }\end{array}$ & 1 & -1 & 1 & I & 1 & 1 & $\stackrel{m}{a}$ & 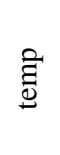 & $\begin{array}{l}\frac{1}{p} \\
\frac{1}{\omega}\end{array}$ &.$\Xi$ & $-r$ & के & $\hat{0}$ & t & $\frac{\mathscr{v}}{\pi}$ \\
\hline $\begin{array}{l}\text { Pinnularia } \\
\text { viridis } \\
\text { Ehrenberg } \\
\text { (Nitzsch) }\end{array}$ & 1 & 1 & 1 & I & 1 & $\dashv$ & â & 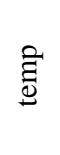 & $\begin{array}{l}\frac{1}{5} \\
\dot{1} \\
\omega\end{array}$ &.$\Xi$ & $\cdot-$ & 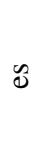 & $\dddot{m}$ & $\begin{array}{l}0 \\
\vdots\end{array}$ & 营 \\
\hline $\begin{array}{l}\text { Placoneis } \\
\text { amphibola } \\
\text { (Cleve) } \\
\text { Cox E. J. }\end{array}$ & 1 & 1 & 1 & 1 & 1 & -1 & 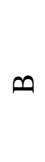 & $\overrightarrow{0}$ & है &.$\Xi$ & $-r$ & 1 & $\stackrel{-}{\circ}$ & 录 & $\frac{\mathscr{y}}{\tilde{\sigma}}$ \\
\hline $\begin{array}{l}\text { Placoneis } \\
\text { exigua } \\
\text { (Gregory) } \\
\text { Mereschko- } \\
\text { vsky }\end{array}$ & -1 & 1 & 1 & I & 1 & 1 & $m$ & 1 & 1 &. $\bar{g}$ & -7 & $\mathscr{0}$ & $\stackrel{+}{-}$ & है & 1 \\
\hline $\begin{array}{l}\text { Placoneis } \\
\text { placentula } \\
\text { (Ehrenberg) } \\
\text { Mereschko- } \\
\text { wsky }\end{array}$ & -1 & 1 & 1 & I & 1 & 1 & $\infty$ & 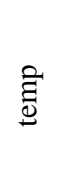 & $\begin{array}{l}\frac{v}{p} \\
\dot{w}\end{array}$ & तै & -7 & w & $\stackrel{\text { ?ִ }}{\text { Tִ }}$ & 0 & $\frac{\mathscr{0}}{\pi}$ \\
\hline
\end{tabular}


Table 2 (continued): Diversity and ecology of diatom algae in the thermal and mineral water sources of Pamir with species autecology. Number of springs 1-6 as in table 1.

\begin{tabular}{|c|c|c|c|c|c|c|c|c|c|c|c|c|c|c|c|}
\hline Таха & -1 & $\sim$ & $m$ & $\nabla$ & مـ & 0 & 蒫 & $H$ & ஷ্் & 兑 & $\overline{\mathbb{I}}$ & D & जิ & 尽 & 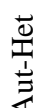 \\
\hline $\begin{array}{l}\text { Planothidium } \\
\text { lanceolatum } \\
\text { (Brébisson } \\
\text { ex Kützing) } \\
\text { Lange- } \\
\text { Bertalot } 1999\end{array}$ & 1 & 1 & I & I & -1 & I & $\frac{m}{n^{\prime}}$ & $\begin{array}{l}\text { E } \\
\text { 营 }\end{array}$ & $\begin{array}{l}5 \\
\frac{1}{1} \\
\frac{1}{\omega}\end{array}$ & $\frac{\Psi}{\pi}$ & $-\rightarrow$ & $\stackrel{\star 凶}{\omega}$ & . & 0 & ثี兀 \\
\hline $\begin{array}{l}\text { Rhopalodia } \\
\text { gibba } \\
\text { (Ehrenberg) } \\
\text { Otto Müller }\end{array}$ & -1 & 1 & 1 & 1 & I & 1 & 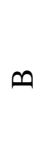 & 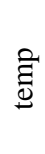 & I & $\frac{\Psi}{\pi}$ & $\rightarrow$ & $\mathscr{\theta}$ & $\stackrel{\nabla}{-}$ & E̦ & I \\
\hline $\begin{array}{l}\text { Rhopalodia } \\
\text { gibba var. } \\
\text { mongolica } \\
\text { (Østrup) } \\
\text { Proschkina- } \\
\text { Lavrenko } \\
\end{array}$ & | & $\neg$ & 1 & I & I & I & 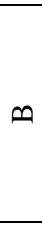 & 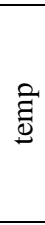 & I & 㫕 & $\rightarrow$ & $\mathscr{E}$ & $\stackrel{\nabla}{-}$ & 1 & 1 \\
\hline $\begin{array}{l}\text { Rhopalodia } \\
\text { gibba var. } \\
\text { ventricosa } \\
\text { (Kützing) } \\
\text { Mayer }\end{array}$ & $\neg$ & 1 & 1 & 1 & 1 & 1 & 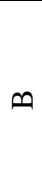 & 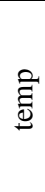 & 1 & $\frac{W}{\pi}$ & $\cdot-1$ & $\mathscr{\theta}$ & $\stackrel{+}{\sim}$ & 1 & 1 \\
\hline $\begin{array}{l}\text { Rhopalodia } \\
\text { gibberula } \\
\text { (Ehrenberg) } \\
\text { Müller O. }\end{array}$ & $\neg$ & I & 1 & 1 & 1 & 1 & 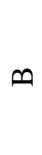 & 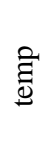 & 离 & $\frac{W}{\pi}$ & हี & $\mathscr{\theta}$ & $\stackrel{\circ}{i}$ & $\stackrel{\mathscr{E}}{\Xi}$ & 1 \\
\hline $\begin{array}{l}\text { Rhopalodia } \\
\text { gibberula var. } \\
\text { producta } \\
\text { (Grunow) } \\
\text { Müller O. }\end{array}$ & | & $\neg$ & 1 & 1 & 1 & I & 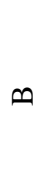 & 1 & है & 㫕 & $I$ & 1 & 1 & 1 & 1 \\
\hline $\begin{array}{l}\text { Rhopalodia } \\
\text { musculus } \\
\text { (Kützing) } \\
\text { Müller O. }\end{array}$ & -1 & 1 & 1 & 1 & 1 & 1 & $\begin{array}{l}n \\
n \\
1 \\
n\end{array}$ & 1 & 离 & 记 & ह & 1 & 요 & 1 & 1 \\
\hline $\begin{array}{l}\text { Rhopalodia } \\
\text { musculus var. } \\
\text { mirabilis } \\
\text { Fricke }\end{array}$ & $\neg$ & 1 & 1 & 1 & 1 & 1 & 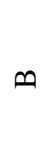 & 1 & 1 & $\widetilde{\pi}$ & $I$ & 1 & $\stackrel{\circ}{i}$ & $\stackrel{\mathscr{E}}{\Xi}$ & 1 \\
\hline
\end{tabular}


Table 2 (continued): Diversity and ecology of diatom algae in the thermal and mineral water sources of Pamir with species autecology. Number of springs 1-6 as in table 1.

\begin{tabular}{|c|c|c|c|c|c|c|c|c|c|c|c|c|c|c|c|}
\hline Таха & -1 & $N$ & $m$ & $\nabla$ & L & 0 & ] & $H$ & ô & $\stackrel{T}{a}$ & 苂 & D & 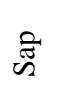 & $\stackrel{8}{9}$ & 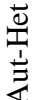 \\
\hline $\begin{array}{l}\text { Rossithidium } \\
\text { anastasiae } \\
\text { (Kaczm.) } \\
\text { Potapova }\end{array}$ & 1 & 1 & 1 & 1 & $-r$ & 1 & $\emptyset$ & 1 & 1 & 1 & 1 & 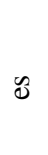 & $\stackrel{\Delta}{\circ}$ & 1 & 1 \\
\hline $\begin{array}{l}\text { Sellaphora } \\
\text { pupula } \\
\text { (Kützing) } \\
\text { Mereschko- } \\
\text { wsky }\end{array}$ & 1 & -1 & 1 & 1 & 1 & 1 & 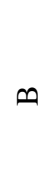 & & $\vec{\omega}$ & $\stackrel{\Xi}{.}$ & $I$ & ฝ & $\stackrel{\rightarrow}{-}$ & छี & $\frac{\mathscr{v}}{\pi}$ \\
\hline $\begin{array}{l}\text { Stauroneis } \\
\text { acuta } \\
\text { Smith W. }\end{array}$ & 1 & 1 & - & 1 & 1 & 1 & 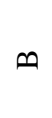 & 1 & $\begin{array}{l}\frac{5}{\omega} \\
\frac{1}{\omega}\end{array}$ & 㫕 & $r$ & 1 & $\stackrel{\circ}{\rightarrow}$ & 疋 & 1 \\
\hline $\begin{array}{l}\text { Stauroneis } \\
\text { anceps } \\
\text { Ehrenberg }\end{array}$ & 1 & 1 & $\neg$ & 1 & 1 & -1 & $\stackrel{m}{\stackrel{m}{a}}$ & 1 & $\begin{array}{l}\frac{y}{p} \\
\frac{\omega}{\omega}\end{array}$ & 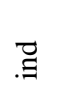 & $r$ & 希 & $\stackrel{m}{-}$ & ₹̦ & 苋 \\
\hline $\begin{array}{l}\text { Staurosira } \\
\text { construens } \\
\text { Ehrenberg }\end{array}$ & 1 & -1 & 1 & 1 & 1 & $r$ & $\stackrel{m}{\prime \prime}$ & 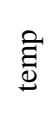 & $\begin{array}{l}\frac{5}{4} \\
\frac{1}{\omega}\end{array}$ & 㫕 & $r$ & 希 & $\stackrel{m}{-}$ & 气̆ & 㫕 \\
\hline $\begin{array}{l}\text { Stephanodiscus } \\
\text { astraea } \\
\text { (Ehrenberg) } \\
\text { Grunow } \\
\text { in Cleve and } \\
\text { Grunow }\end{array}$ & 1 & 1 & $\neg$ & 1 & 1 & - & $a_{1}$ & 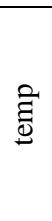 & $\ddot{\omega}$ & नी & $r$ & $\approx$ & مִ & 气ี & 1 \\
\hline $\begin{array}{l}\text { Surirella } \\
\text { angusta } \\
\text { Kützing }\end{array}$ & 1 & -1 & I & 1 & 1 & -1 & $\stackrel{m}{\prime}$ & 1 & $\begin{array}{l}\frac{5}{4} \\
\frac{1}{\omega}\end{array}$ & 㫕 & $r$ & 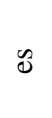 & 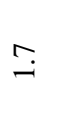 & 0 & है \\
\hline $\begin{array}{l}\text { Surirella } \\
\text { angusta } \\
\text { var. } \\
\text { constricta } \\
\text { Hustedt }\end{array}$ & -1 & 1 & 1 & I & 1 & 1 & 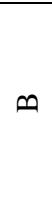 & 1 & 1 & 1 & $r$ & 1 & 1 & 1 & 1 \\
\hline
\end{tabular}


Table 2 (continued): Diversity and ecology of diatom algae in the thermal and mineral water sources of Pamir with species autecology. Number of springs 1-6 as in table 1.

\begin{tabular}{|c|c|c|c|c|c|c|c|c|c|c|c|c|c|c|c|}
\hline Таха & - & $N$ & $m$ & $\nabla$ & in & 0 & శ్తి & $H$ & है & $\stackrel{I}{\mathrm{a}}$ & 苂 & D & लि & $\stackrel{\varrho}{\Theta}$ & 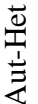 \\
\hline $\begin{array}{l}\text { Surirella } \\
\text { brebissonii } \\
\text { Krammer and } \\
\text { Lange- } \\
\text { Bertalot }\end{array}$ & - & 1 & 1 & 1 & 1 & 1 & $\infty$ & 1 & $\begin{array}{l}\frac{5}{\omega} \\
\frac{1}{\omega}\end{array}$ & 㫕 & $-r$ & 1 & $\stackrel{\sim}{-}$ & 1 & 1 \\
\hline $\begin{array}{l}\text { Surirella } \\
\text { linearis } \\
\text { Smith W. }\end{array}$ & - & 1 & 1 & I & 1 & 1 & $\stackrel{m}{a}$ & 1 & 1 &.$\Xi$ & $\rightarrow$ & 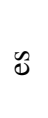 & $\stackrel{100}{0}$ & छั & 1 \\
\hline $\begin{array}{l}\text { Surirella } \\
\text { linearis var. } \\
\text { helvetica } \\
\text { (Brun) } \\
\text { Meister }\end{array}$ & 1 & - & 1 & I & 1 & 1 & $\infty$ & 1 & 1 & $\underset{\Xi}{\Xi}$ & $-r$ & $\mathscr{\Xi}$ & $\stackrel{L}{0}$ & ठ & 1 \\
\hline $\begin{array}{l}\text { Surirella } \\
\text { minuta } \\
\text { Brébisson } \\
\text { ex Kützing }\end{array}$ & - & 1 & 1 & I & I & 1 & 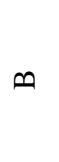 & 1 & 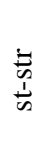 &.$\Xi$ & $-r$ & $y$ & $\stackrel{\infty}{\rightarrow}$ & $\stackrel{\Xi}{\Xi}$ & I \\
\hline $\begin{array}{l}\text { Synedra } \\
\text { montana } \\
\text { Krasske ex } \\
\text { Hustedt }\end{array}$ & 1 & $\dashv$ & 1 & 1 & 1 & 1 & $\infty$ & 1 & 1 & 1 & 1 & 1 & 1 & 1 & 1 \\
\hline $\begin{array}{l}\text { Synedra } \\
\text { rumpens } \\
\text { Kützing }\end{array}$ & 1 & $\neg$ & 1 & 1 & 1 & 1 & $\infty$ & 1 & 1 & 㫕 & $-r$ & $\mathscr{D}$ & 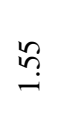 & 1 & 1 \\
\hline $\begin{array}{l}\text { Synedra } \\
\text { goulardii } \\
\text { Brébisson ex } \\
\text { Cleve and } \\
\text { Grunow }\end{array}$ & 1 & -1 & 1 & 1 & 1 & 1 & $\infty$ & 1 & 1 & 1 & 1 & 1 & 1 & 1 & 1 \\
\hline $\begin{array}{l}\text { Tabularia } \\
\text { fasciculata } \\
\text { (Agardh C.) } \\
\text { Williams D. } \\
\text { M. and Round }\end{array}$ & $r$ & 1 & 1 & 1 & 1 & 1 & $\stackrel{m}{a}$ & 1 & $\vec{n}$ &.$\Xi$ & 春 & 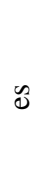 & $\stackrel{\llcorner}{\mathrm{N}}$ & 0 & $\frac{\pi}{\pi}$ \\
\hline $\begin{array}{l}\text { Tetracyclus } \\
\text { rupestris } \\
\text { (Kützing) } \\
\text { Grunow in } \\
\text { Van Heurck }\end{array}$ & - & 1 & 1 & I & 1 & 1 & $\stackrel{m}{a}$ & $\overline{8}$ & $\mathbb{\sigma}$ & $\underset{\widetilde{d}}{\breve{d}}$ & $-r$ & 1 & $\stackrel{\infty}{0}$ & ठ & 1 \\
\hline
\end{tabular}


Table 2 (continued): Diversity and ecology of diatom algae in the thermal and mineral water sources of Pamir with species autecology. Number of springs 1-6 as in table 1.

\begin{tabular}{|c|c|c|c|c|c|c|c|c|c|c|c|c|c|c|c|}
\hline Таха & $\neg$ & $N$ & $m$ & $\nabla$ & 10 & 0 & 茎 & 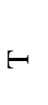 & ôे & 壳 & 茎 & D & ज्ञీ & 怘 & 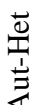 \\
\hline 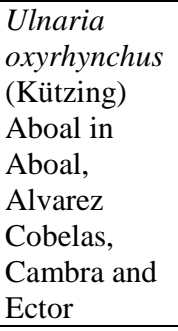 & $\neg$ & 1 & 1 & | & 1 & 1 & $\frac{\infty}{1}$ & 1 & 1 & त्ठ & $\rightarrow$ & $\mathscr{Q}$ & $\stackrel{+}{v}$ & ڤ్ & 1 \\
\hline Total: & 92 & 48 & 18 & 14 & 9 & 18 & & & & & & & & & \\
\hline \multicolumn{16}{|c|}{$\begin{array}{l}\text { Note. Substrate preferences (Hab): P - planktonic, P-B - plankto-benthic, B - benthic, S - soil, Ep - epiphyte. } \\
\text { Temperature preferences (T): temp - temperate temperature, eterm - eurythermic, warm - warm-water, cool - } \\
\text { could water inhabitants. Oxygenation and streaming (Oxy): st - standing water, str - streaming water, st-str - low } \\
\text { streaming water, ae - aerophiles. Halobity degree (Hal): i - oligohalobes-indifferent, hl - halophiles, hb - } \\
\text { halophobes, mh - mesohalobes. Acidity degree (pH): alf - alkaliphiles, ind - indifferents; acf - acidophiles, alb - } \\
\text { alkalibiontes. Organic pollution indicators according to Watanabe (D): sx - saproxenes, es - eurysaprobes, sp - } \\
\text { saprophiles. Species-specific index of saprobity S (Sap). Nitrogen uptake metabolism (Aut-Het): ats - nitrogen- } \\
\text { autotrophic taxa, tolerating very small concentrations of organically bound nitrogen; ate - nitrogen-autotrophic } \\
\text { taxa, tolerating elevated concentrations of organically bound nitrogen; hne - facultatively nitrogen-heterotrophic } \\
\text { taxa, needing periodically elevated concentrations of organically bound nitrogen. Trophic state (Tro): ot - } \\
\text { oligotraphentic; o-m - oligo-mesotraphentic; m - mesotraphentic; me - meso-eutraphentic; e - eutraphentic; o-e } \\
\text { - oligo-eutraphentic. }\end{array}$} \\
\hline
\end{tabular}

We analyzed bioindicator species representation in each studied source as well as distribution of specific groups over source communities and between them. The bioindication histograms are organized in order of water $\mathrm{pH}$ decreasing. Table 2 and figure 4 shows that benthic species prevail in all studied sources but Garm-Chashma and Barshor were slightly enriched by planktonic inhabitants because only two of these sources have some water mass (Barinova and Niyatbekov, 2017). Temperature species-indicators were represented as whole small taxa, but it also shows prevalence of temperate temperature water inhabitants, a number of which are increased with decreasing of water alkalinity. In figure 4, we have demonstrated that in the Sist community, the presence of warm-water species was high but it is not true because this community contains only nine taxa and we found only one temperature indicator which is related to warm-water group.

Enrichments of water with oxygen is a very important characteristic of aquatic ecosystem because without it cannot exist as a biotic part of the ecosystem. Bioindication groups were mostly related to slow moving or standing water that is in small water sources that we study. In the second place we studied, there were indicators of streaming waters that reflect output of water from the sources with small streams. Standing water indicators are slightly increased with decreasing water alkalinity but it can be more related to the water volume of the source. In the Sist we found only two groups related to water moving and oxygenation but it is difficult to assess because the species richness of diatom indicators is represented by nine taxa only. 


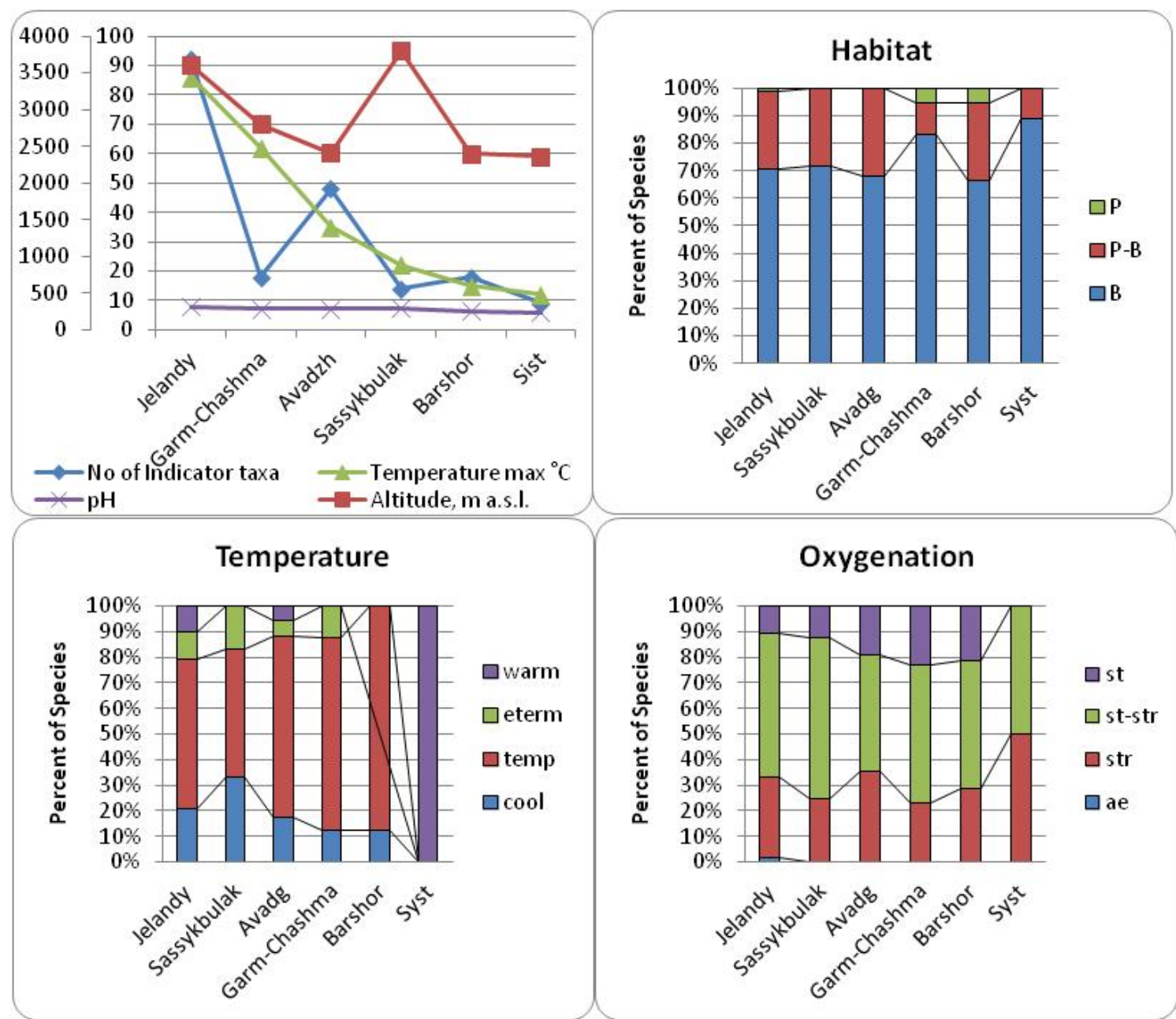

Figure 4: Water variables and indicator species of substrate preferences, temperature, and oxygenation distribution in the studied sources of Pamir.

Water $\mathrm{pH}$ bioindication shows (Fig. 5) prevailing alkalic waters over the studied sources although water $\mathrm{pH}$ is slightly decreased from 7.8 in Jelandy to 5.4 in Sist. As a whole, indicator groups of alkaliphiles and indifference take about $90 \%$ of indicator species in each source community. We assume that the community of each source has some buffered property for stabilizing source environment, and give the possibility to survive for species with broad ecological amplitude. The same situation can be seen in bioindication of water salinity. Figure 5 shows the prevalence of indifferent group and insufficient numbers of the low- and highsalinity indicator number. 


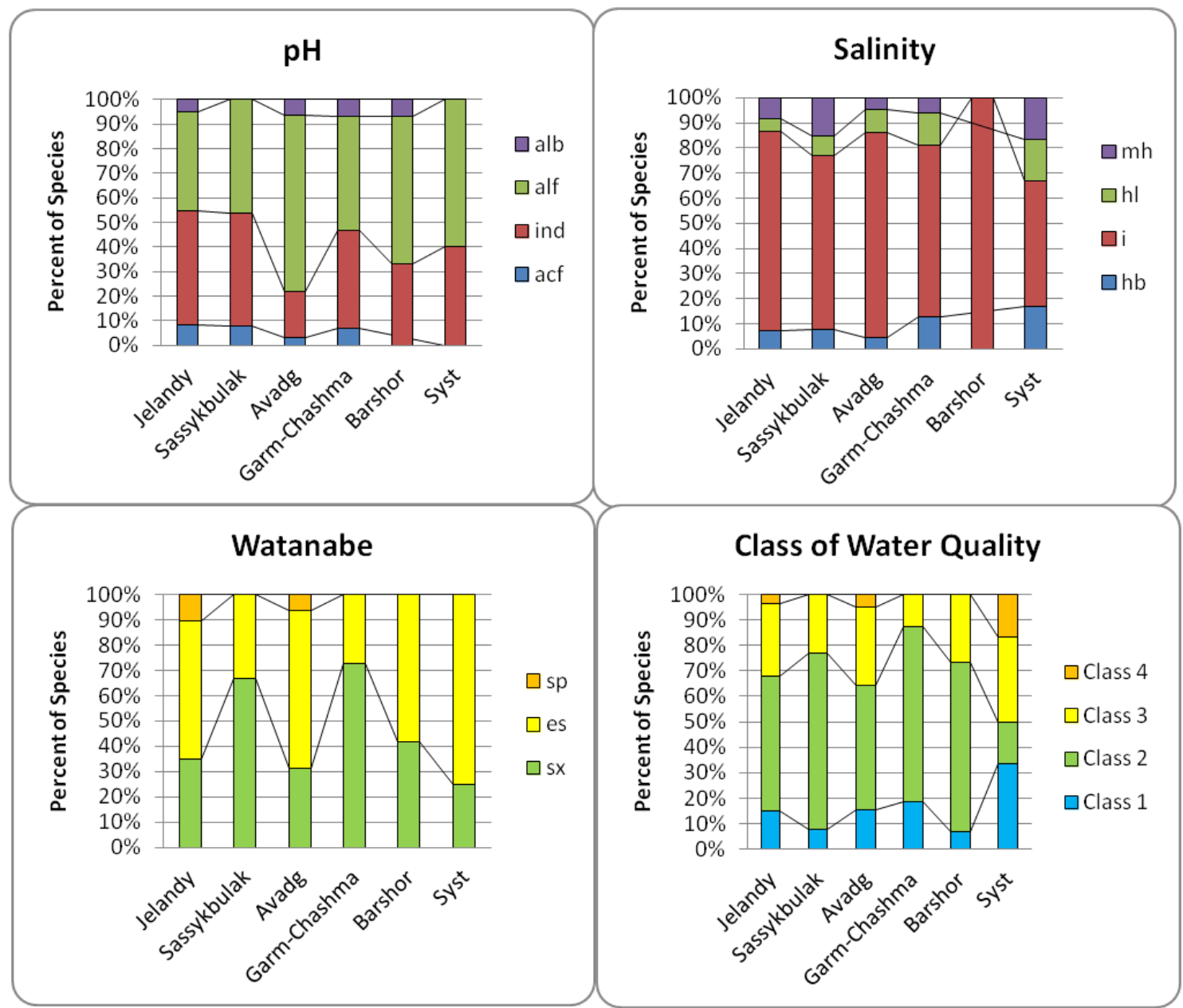

Figure 5: Indicator species of water $\mathrm{pH}$, salinity, and organic pollution with Water Quality Classes distribution in the studied sources of Pamir.

Studied thermal and mineral springs are represented by natural protected areas, therefore, most important to reveal anthropogenic influence on its communities as criteria of protection effectiveness. Figure 5 represents bioindication results of organic pollution with the Watanabe system as well as with the Sládeček's system. The source communities are represented by all indicator groups of Watanabe's method but number of taxa of clear (sx) and middle polluted (es) indicators are prevalent. The colours of groups are the same as are used in the EU system. Classes 1 and 2 indicators in Sládeček system contain most parts of the community in each studied source. That means that water sources are organically clear. Even indicators of middle pollution Class 3 were not as large in number as Class 2. Only few species of Class 4 were found but indicators of polluted water Class 5 were not revealed at all. All this led us to conclude that studied sources are represented by natural unpolluted waters with natural undisturbed aquatic ecosystems. 
The effectiveness of photosynthesis was determined in each of the sources with help of the nutrition type indicator species distribution. The photosynthetic diatoms are prevail (97\%) in communities of all studied sources (Fig. 6). Organic pollution of sources is related to trophic level of its ecosystem and shows (Fig. 6) that communities of each source are represented by different groups of indicators of trophic state. In the trend of increasing trophic level from oligotrophic indicators (ot) to eutrophic (e and o-e) can be seen that most part of indicators was related to oligo- and mesotrophic groups in each source. Eutrophic groups were represented by about $20 \%$ of indicators in Jelandy, Avadg, and Barshor sources. We can see eutrophic groups as half of indicators in the Sist only, but can be keeping in mind that all species in Sist are represented by nine taxa. Therefore, ecosystems of studied sources can be characterized as the oligo-mesotrophic level that usually is in the unpolluted protected areas.

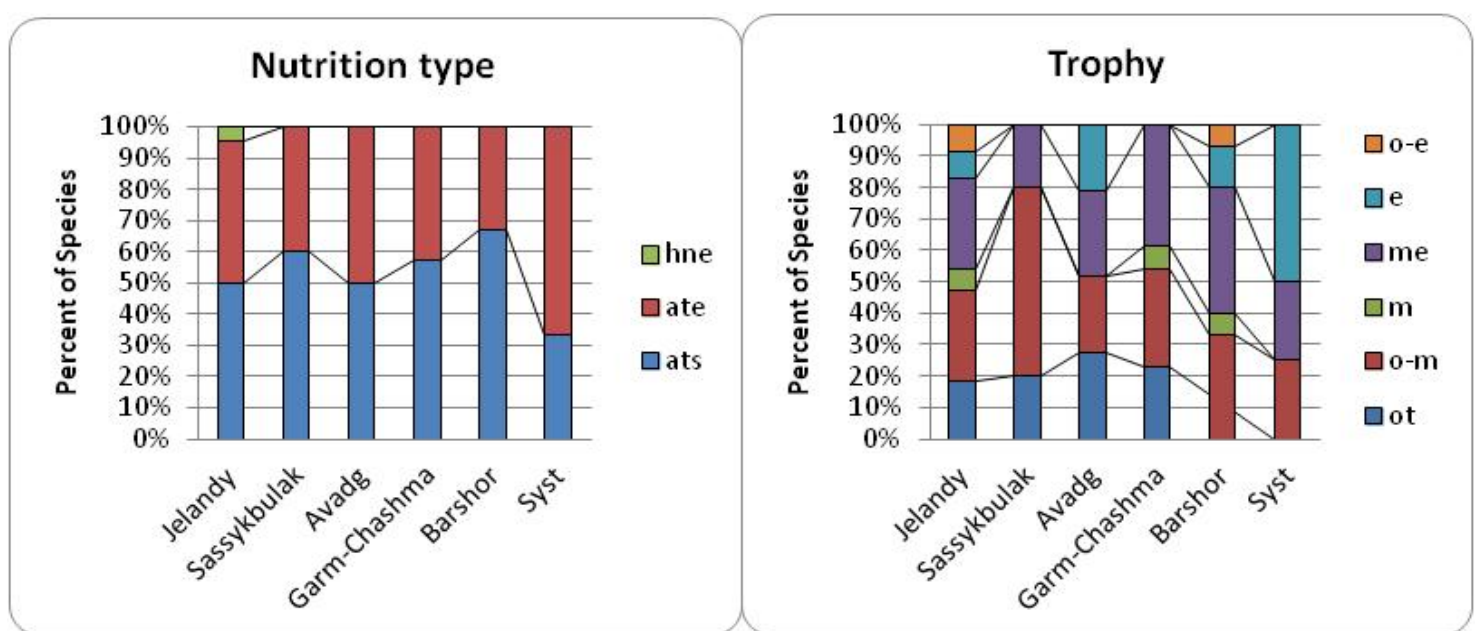

Figure 6: Distribution of indicator species of algal nutrition type and trophic level of aquatic ecosystem in the studied thermal and mineral sources of Pamir.

\section{CONCLUSIONS}

Algae diversity study in the sources of the Pamir Mountains is far from complete, but it is very important because thermal and mineral springs in Pamir are represented by diversity in the natural protected areas. Bioindication of diverse variables in six studied sources help us to characterize the environmental variables that have not yet been measured on these hard-toreach sources. We implemented bioindication approach for the first time for Pamir sources ecosystem assessment. The results can confirm or disprove the applicability of bioindication methods for such different source waters. This first experience can help to characterize the effectiveness of natural conservation and protection mechanisms for Pamir protected areas.

Bioindication on the base of 166 revealed taxa of diatom algae characterize six studied sources environment as low-alkaline, well oxygenated waters with low salinity, and low to middle organically polluted in which diatom community preferred to survive as periphytonic and benthic occupants that preferred temperate temperature waters and photosynthetic way of protein synthesis. The trophic level of each studied source was assessed for the first time, and the data revealed that all of them have a state from oligotrophic to mesotrophic. These results can confirm the effectiveness of a nature conservation system in Tajikistan as well as the relevance of bioindication methods used to assess and monitor water sources on the protected territories. 


\section{ACKNOWLEDGEMENTS}

The work was partly supported by the Israeli Ministry of Absorption. 


\section{REFERENCES}

1. Barinova S. and Niyatbekov T. P., 2017 - Algal Diversity of the Pamir High Mountain Mineral Springs in Environmental Variables Gradient, International Journal of Environmental Sciences and Natural Resources, 7, 2, 555706, DOI: 10.19080/IJESNR.2017.07.555706.

2. Barinova S. S., Medvedeva L. A. and Anissimova O. V., 2006 - Diversity of algal indicators in environmental assessment, Tel Aviv, Pilies Studio, 458. (in Russian)

3. Bokhodjaev I. Y. and Davlatmamadov S. M., 1994 - Medical mineral springs of Pamir, Dushanbe, 123. (in Russian)

4. Churshina N. M., 1982 - Mineral, thermal and cold water, in Tajikistan (nature and natural resources), Dushanbe, 125-131.

5. Fránková M., Bojková J., Pouličková A. and Hájek M., 2009 - The structure and species richness of the diatom assemblages of the Western Carpathian spring fens along the gradient of mineral richness, Fottea, 9, 2, 355-368.

6. Leira M., Meijide-Falide R. and Torres E., 2017 - Diatom communities in thermo-mineral springs of Galicia (NW Spain), Diatom Research, 32, 1, 29-42, doi.org/10.1080/0269249X.2017.1286266.

7. Sinitean A. and Kutaşi R., 2012 - A study of the benthic diatom flora of the Cefa Nature Park (Crişana, Romania), Transylvanian Review of Systematical and Ecological Research, 13, Wetlands Diversity, 1-10.

8. Sisma-Ventura G., Barinova S., Greenbaum N. and Tavassi M., 2010 - The influence of low storm discharge on nutrients and algal periphyton dynamics of a small Mediterranean, mountainous, ephemeral stream pond - Oren River basin, Carmel Mountains (Israel), Transylvanian Review of Systematical and Ecological Research, 10, Wetlands Diversity, 137150.

9. Torrisi M. and Dell'uomo A., 2012 - The diatoms of the Marmore Waterfall (Umbria, Italy), Transylvanian Review of Systematical and Ecological Research, 14, Wetlands Diversity, 33-46.

10. Waring G. A., 1965 - Thermal springs of the United States and other countries of the world. A summary, Geolgical Survey Professional Paper, 492, Washington, United States Government Printing Office, 400.

11. Winterbourn M. J., 1969 - The distribution of algae and insects in hot spring thermal gradients at Waimangu, New Zealand, New Zealand Journal of Marine and Freshwater Research, 3, 3, 459-465, DOI: 10.1080/00288330.1969.9515311. 